ARTICLE

https://doi.org/10.1038/s41467-019-10274-1

\title{
The AvrPm3-Pm3 effector-NLR interactions control both race-specific resistance and host-specificity of cereal mildews on wheat
}

Salim Bourras (10) 1,2,7, Lukas Kunz (1) 1,7, Minfeng Xue 3,4,5,7, Coraline Rosalie Praz (iD 1, Marion Claudia Müller (1) 1, Carol Kälin (1) ${ }^{1}$, Michael Schläfli ${ }^{1}$, Patrick Ackermann ${ }^{1}$, Simon Flückiger (1) ${ }^{1}$, Francis Parlange ${ }^{1}$, Fabrizio Menardo ${ }^{1}$, Luisa Katharina Schaefer ${ }^{1}$, Roi Ben-David ${ }^{6}$, Stefan Roffler ${ }^{1}$, Simone Oberhaensli ${ }^{1}$, Victoria Widrig (i) ${ }^{1}$, Stefan Lindner (10 1, Jonatan Isaksson ${ }^{1}$, Thomas Wicker ${ }^{1}$, Dazhao $\mathrm{Yu}^{3,4,5} \&$ Beat Keller (i) ${ }^{1}$

The wheat Pm3 resistance gene against the powdery mildew pathogen occurs as an allelic series encoding functionally different immune receptors which induce resistance upon recognition of isolate-specific avirulence (AVR) effectors from the pathogen. Here, we describe the identification of five effector proteins from the mildew pathogens of wheat, rye, and the wild grass Dactylis glomerata, specifically recognized by the PM3B, PM3C and PM3D receptors. Together with the earlier identified AVRPM3 ${ }^{A 2 / F 2}$, the recognized AVRs of $\mathrm{PM} 3 \mathrm{~B} / \mathrm{C}$, (AVRPM3 ${ }^{\mathrm{B} 2 / \mathrm{C} 2}$ ), and PM3D (AVRPM3 ${ }^{\mathrm{D} 3}$ ) belong to a large group of proteins with low sequence homology but predicted structural similarities. AvrPm3 $3^{62 / c 2}$ and $A v r P m 3^{d 3}$ are conserved in all tested isolates of wheat and rye mildew, and non-host infection assays demonstrate that $\mathrm{Pm} 3 \mathrm{~b}, \mathrm{Pm} 3 \mathrm{c}$, and $\mathrm{Pm} 3 \mathrm{~d}$ are also restricting the growth of rye mildew on wheat. Furthermore, divergent AVR homologues from non-adapted rye and Dactylis mildews are recognized by $\mathrm{PM} 3 \mathrm{~B}, \mathrm{PM} 3 \mathrm{C}$, or $\mathrm{PM} 3 \mathrm{D}$, demonstrating their involvement in host specificity.

\footnotetext{
${ }^{1}$ Department of Plant and Microbial Biology, University of Zurich, 8008 Zurich, Switzerland. ${ }^{2}$ Department of Forest Mycology and Plant Pathology, Division of Plant Pathology, Swedish University of Agricultural Sciences, 75007 Uppsala, Sweden. ${ }^{3}$ Institute of Plant Protection and Soil Science, Hubei Academy of Agricultural Sciences, Wuhan 430064, China. ${ }^{4}$ Ministry of Agriculture Key Laboratory of Integrated Pest Management in Crops in Central China, Wuhan 430064, China. ${ }^{5}$ College of Life Science, Wuhan University, Wuhan 430072, China. ${ }^{6}$ Institute of Plant Science, ARO-Volcani Center, 50250 Bet Dagan, Israel. ${ }^{8}$ These authors contributed equally: Salim Bourras, Lukas Kunz, Minfeng Xue. Correspondence and requests for materials should be addressed to $S$. B. (email: salim.bourras@slu.se) or to D.Y. (email: dazhaoyu@china.com) or to B.K. (email: bkeller@botinst.uzh.ch)
} 
F ungi are highly adaptive, widespread organisms and ubiquitous pathogens of animals and plants responsible for population declines and pandemics in natural and agricultural ecosystems ${ }^{1-5}$. In wheat, there have been two recent fungal pandemics caused by the emergence of wheat blast ${ }^{6}$ and stem rust $\left(\mathrm{Ug} 99^{7}\right)$. The Sr35 resistance $(R)$ gene encodes a nucleotide bindingleucine rich repeat (NLR) immune receptor that upon recognition of the avirulence protein AVRSr35 confers near-immunity to Ug998,9. This case demonstrates that effector-NLR interactions conferring immunity following the simplest model of $A v r-R$ genefor-gene interactions ${ }^{10,11}$ provide an important leverage for achieving rapid control of emerging disease pandemics in crops.

Powdery mildews are agronomically important fungal pathogens of large numbers of wild and cultivated species. In cereals, the powdery mildew disease is caused by a single species, Blumeria graminis. It occurs in different, highly host-specific forms called formae speciales ${ }^{12,13}$. For example, B. graminis f. sp. tritici (B.g. tritici) exclusively grows on wheat, whereas B. graminis f. sp. secalis (B.g. secalis) and B. graminis f. sp. dactylidis (B.g. dactylidis) specifically infect rye and the wild grass species Dactylis glomerata, respectively ${ }^{13}$. Powdery mildews are obligate biotrophs, growing and reproducing exclusively on living host tissue. This requires suppression of host immune responses which is possibly achieved through the massive secretion of small virulence proteins called effectors ${ }^{14,15}$. Recent studies in barley powdery mildew have established the role of some effectors as virulence factors ${ }^{16-21}$. Effector proteins can be specifically recognised by NLR plant immune receptors whose activation confer resistance to the invading pathogen. This isolate-specific resistance is often linked to a rapid cell death response at sites of attempted infection, designated as the hypersensitive response $(\mathrm{HR})^{22,23}$. Effectors recognised by R-proteins are called avirulence (AVR) proteins and are often found to be polymorphic in different isolates of the pathogenic fungus ${ }^{15}$.

Allelic series of NLR-type receptors have been described in several plant species. The largest allelic series of resistance genes in wheat is formed by the $P m 3$ gene against powdery mildew. Up to date, 17 functional alleles (Pm3a-g; Pm3k-Pm3t) have been cloned and functionally validated 24,25 , and they confer complete resistance against distinctly different sets of B.g. tritici races ${ }^{26}$. Compared to other well studied allelic series of $R$ genes such as the flax rust (Melampsora lini) resistance gene $L$ or the B.g. hordei resistance gene $M l a$, the $P m 3$ series stands out due to its very high $(>97 \%)$ sequence identity on the protein level $22,25,27$.

The molecular basis of the functional diversity of the closely related Pm3 alleles is largely unknown. Up to date, the only known avirulence gene for a $P m 3$ allele is $A v r P m 3^{a 2 / f 2}$, an effector gene recognised by the $P m 3 a$ and $P m 3 f$ alleles ${ }^{28}$. Despite high sequence conservation among the Pm3 NLRs, no allele other than $P m 3 a$ and $P m 3 f$ can recognise $A v r P m 3^{a 2 / f 2}$ or any of its closest gene family relatives or natural haplotype variants ${ }^{28,29}$. Furthermore Bourras and collaborators ${ }^{28}$ provided evidence that the $P m 3$ resistance follows a genetically complex gene-for-gene model ${ }^{11}$, which involves a pathogen encoded suppressor of avirulence $\left(S v r P m 3^{a 1 / f 1}\right)$ acting on several AvrPm3-Pm3 specificities.

In this study, we describe the identification of the $A v r P m 3^{b 2 / c 2}$ and $A v r P m 3^{d 3}$ genes and provide molecular evidence that specificity of the Pm3 NLRs is based on recognition of highly sequence diverse, but structurally similar effectors. We also demonstrate that the same effector genes are conserved in the non-adapted rye and Dactylis powdery mildews, thus demonstrating that the Pm3 NLRs, apart from their race-specific resistance function, are potent determinants of host-specificity for grass mildews. Therefore, we propose that the AvrPm3-Pm3 interactions provide a unique model system to understand how NLRs can contribute to both host and non-host resistance.

\section{Results}

Identification of $A v r P m 3$ candidates by effector benchmarking. In a first approach to identify $A v r P m 3$ candidate genes we established a new assay based on the hypothesis that effector proteins acting as AVR factors are likely to share structural similarities, sequence polymorphism, and similar expression patterns at the haustorial stage. At the time this assay was designed, four mildew Avrs were cloned: AvrPm3 $3^{a 2 / f 2}, A v r P m 2$ from B.g. tritici ${ }^{28,30}$ and $A v r_{a 1}, A v r_{a 13}$ from B.g. hordei ${ }^{31}$. They are all encoded by typical effector proteins, ranging in size from 118 $\left(\mathrm{AVR}_{\mathrm{A} 1}\right)$ to 130 aa $\left(\mathrm{AVRPM} 3^{\mathrm{A} 2 / \mathrm{F} 2}\right)$, with a predicted $\mathrm{N}$ terminal signal peptide, two conserved cysteine residues (except $A V R_{A 13}$ which contains only one), and high expression at the haustorial stage $^{28,30,31}$. Therefore, we hypothesised that mildew AVRs correspond to short proteins of ca. 120 aa on average, encoded by highly expressed candidate effector genes, that can be differentiated in mildew isolates with contrasting virulence patterns based on sequence polymorphism or gene expression levels.

We scanned the genomes of the three wheat powdery mildew reference isolates, Bgt_96224, Bgt_94202 and Bgt_JIW2, which are polymorphic for several AvrPm3 specificities ${ }^{28,32}$, and systematically classified and scored 580 effectors based on sequence polymorphism (SNPs and deletions), presence of a functional signal peptide, cysteine content, native protein size, and gene expression levels (Supplementary Note 1, Supplementary Fig. 1). This resulted in an "effector benchmarking" list with effector scores for candidate $A v r P m 3$ genes ranging from -8 for the worst candidate $(B g t E-5642)$ to +18 for the two best ones (Bgt_avrF2_9 and BgtE-20041) (Supplementary Note 1, Supplementary Data 1, Supplementary Fig. 1). AvrPm2 and AvrPm3 $3^{a 2 / f 2}$ were used as a control for assessing $A v r$ candidate scoring, and they ranked in the top 20, with a total score of +17 and +16 , respectively. We selected all effector genes scoring at least +10 (i.e. 100 candidates) for further functional validation (Supplementary Data 1).

Identification of $A v r P m 3$ candidates using GWAS. In a second approach to identify $A v r P m 3^{b}, A v r P m 3^{c}$ and $A v r P m 3^{d}$ candidates, we sequenced 72 additional wheat powdery mildew genomes to complete a GWAS (genome wide association study) mapping population of 100 races originating exclusively from China (Supplementary Data 2). Genetic association between sequence polymorphisms and differences in virulence/avirulence patterns on Pm3b and Pm3d was assessed using the Genome Association and Prediction Integrated Tool (GAPIT) ${ }^{33}$. We found no significant association for $A v r P m 3^{d}$. We found significantly associated SNPs for $A v r P m 3^{b}$ on chromosome $5^{34}$, with a peak at position 18,860,696 (Fig. 1a, b). This region overlaps with the physical position of Locus_3, a genetic locus that has been previously described as encoding for the $A v r P m 3^{b 2}$ and $A v r P m 3^{c 2}$ specificities $^{28}$ (Supplementary Note 2). The position of the $A v r P m 3^{b 2}$ peak was located within the genetic interval defined by the Locus_3 flanking markers M049LE and ctg118_21 in the powdery mildew consensus genetic map ${ }^{28}$ (Supplementary Fig. 2).

We used a combination of resources to thoroughly annotate this genetically complex locus (Supplementary Note 2). We found in total four candidate effector genes in the locus: Bgt-51460, $B g t E-20002$, Bgt-55150 and BgtE-ng2, all members of the effector gene family E018 ${ }^{34}$ (Supplementary Fig. 3). Bgt-51460 is not expressed and not polymorphic between the $P m 3 b / c$ avirulent Bgt_96224, and the Pm3b/c virulent Bgt_94202 parents. BgtE20002 is not polymorphic between the isolates Bgt_96224 and Bgt_94202, and it was also identified as a candidate Avr by the benchmarking approach with an overall score of +12 


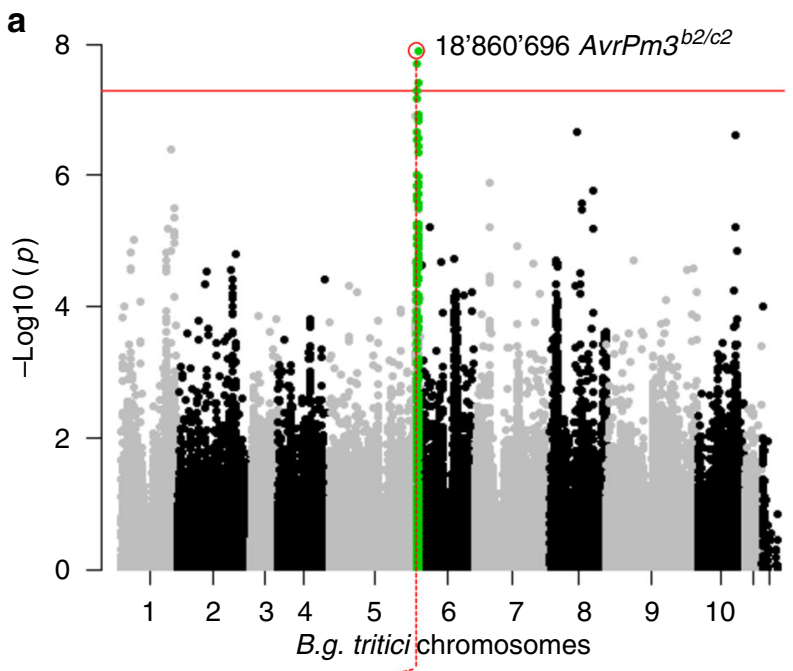

b

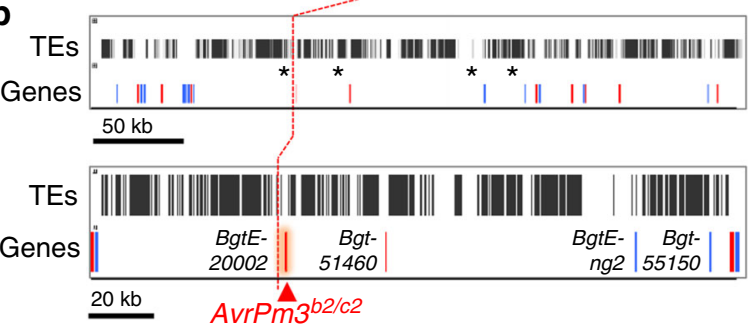

C

>BgtE-20002 (Isolate Bgt_96224)
>BgtE-20002 (Isolate Bgt_94202)
>BgtE-20002_I (Isolate Bgt_07237)

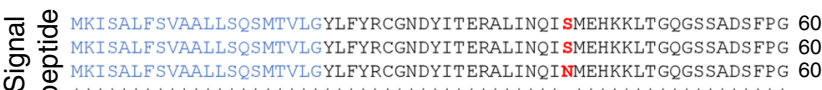

GRATAEVTFWEPSI SN PGTYLDI KVKFDIYROMLSFEVSSSGKRI PCEGDYGAEI PEEDI 120 GRATAEVT FWEPS I SN PGTYLDI KVKFDIYRQMLS FEVSSSGKRI PCEGDYGAEI PEEDL 120 GRATAEVTFWEPSI SN PGTYLDI KVKFDIYROMLSFEVSSSGKRI PCEGDYGAEI PEEDL 120

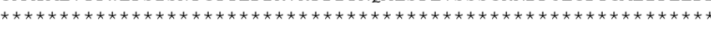

EVSDEPYYAN* 130

EVSDEPYYAN* 130

EVSDEPYYAN* 130

EVSDEPYYAN*

d

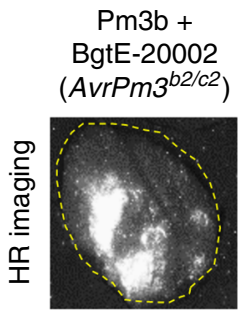

$\mathrm{Pm} 3 \mathrm{~b}+$ BgtE-20002 $\left(A v r P m 3^{b 2 / c \overline{2}}\right)$

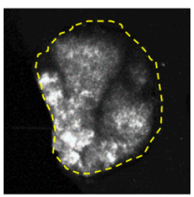

$\mathrm{Pm} 3 \mathrm{c}+$

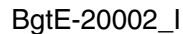

$\left(\right.$ AvrPm3 $\left.^{b 2 / c 2}\right)$

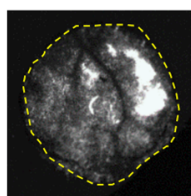

e

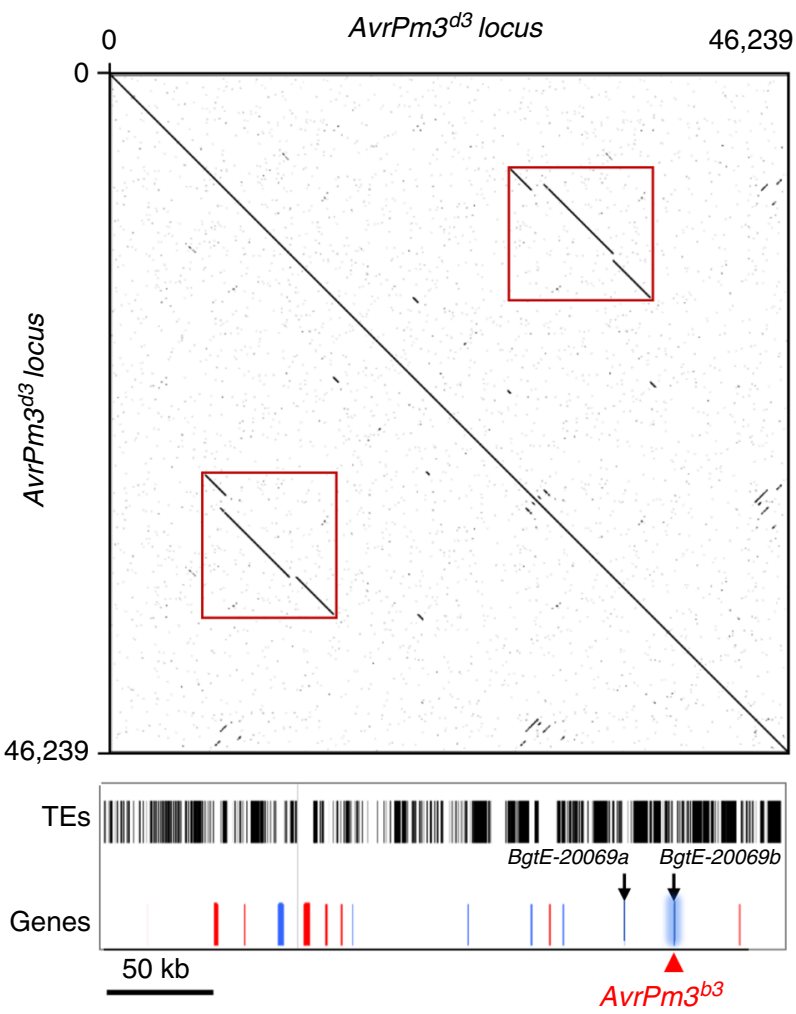

g

>BgtE-20069b_96224

$>$ BgtE-20069a_96224

>BgtE-20069a_94202

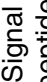

MKIVLPHLAIALIS SVAPALAVI FDCSGVI INGDVVRRVQATGNRAS ISKSSFI HGDSTR 60 MKIVLPHLAIALIS IVAPALAVI FDCSGVI INGDVVRRVQATGNRA.SISKSSSI HGDSTR 60 MKIVLPHLAIALISIVAPALAVI FDCSGVI INGDVVRRVQATGNRA.SISKSSSI HGDSTR 60

TYEYFDIHPNGOYSRDYTGFACFSDTSLPKPDVREHREDAWWPCSSSVI * 109 TYEYFDIHPNGQYSRDYTGFACFSDISLPKPDVREHREDAWWPCSSSVI* 109 TYEYFDI HPNGQYSKDYTGFACFSDI SLPKPDVREHREDAWWPCSSSVI* 109

h

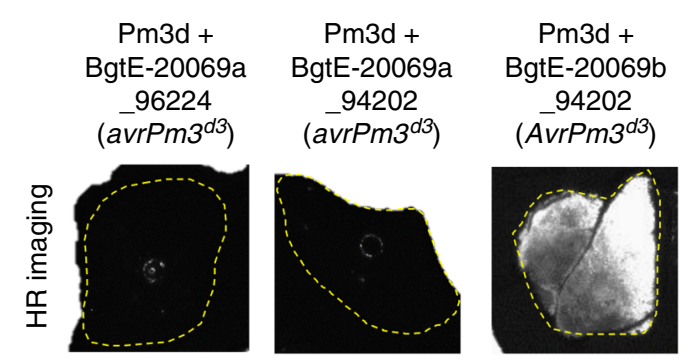

on the GWAS results, genetic information from the powdery mildew consensus map ${ }^{28}$, and thorough annotation of Locus_3, we selected Bgt-51460, BgtE-20002, BgtE-20002_I, Bgt-55150a, $B g t-55150 b$ and $B g t E-n g 2 b$ for further functional validation (Supplementary Note 2, Supplementary Data 3 and 4).

Functional validation of $A v r P m 3^{b 2 / c 2}$ and $A v r P m 3^{d 3}$. To functionally validate the $A v r P m 3$ candidates identified by effector benchmarking and GWAS, we took advantage of our well established agrobacterium-infiltration assay in N. benthamiana ${ }^{28-30}$ allowing transient, heterologous, co-overexpression of effector and 
Fig. 1 Identification and functional validation of $A v r P m 3^{b 2 / c 2}$ and $A v r P m 3^{d 3}$. a-d Identification and functional validation of $A v r P m 3^{b 2 / c 2}$. a GWAS based on 26 avirulent and 74 virulent isolates on $\mathrm{Pm} 3 b$. The peak for $A v r P m 3^{b / c}$ on chromosome 5 is indicated in green. $\mathbf{b}$ TE content of the $A v r P m 3^{b 2 / c 2}$ locus is indicated (black bars), gene content in blue for polymorphic genes between Bgt_96224 and Bgt_94202 and red for non-polymorphic genes. The four effector genes selected for functional validation are indicated with asterisks (upper panel) and labelled in the lower panel. Position of the GWAS peak is indicated (dotted red line). The functionally validated AvrPm3b2/c2 gene is highlighted. c Alignment of the AVRPM3 ${ }^{B 2 / C 2}$ protein sequence encoded by BgtE-20002 in the isolates Bgt_96224, Bgt_94202 and Bgt_07237. The sequence corresponding to the signal peptide (blue), and polymorphic residues (red) are indicated. d Functional validation of the AvrPm3 $3^{62} / c_{-}-P m 3 b / c$ interactions using transient expression assays in $N$. benthamiana. HR was scored 4-5 days after co-infiltration (Fusion-FX imager). e-h identification and functional validation of $A v r P m 3^{d 3}$. e Dot-plot depiction of the duplicated region on chromosome 9 (red frame) from isolate Bgt_96224, containing AvrPm3d3 (BgtE-20069b) and its paralog BgtE-20069a. f Relative position of the BgtE$20069 a$ and BgtE-20069b in the AvrPm3d locus. TE content is indicated (black bars), polymorphic genes between Bgt_96224 and Bgt_94202 are indicated in blue and non-polymorphic in red. Functionally validated $A v r P m 3^{d 3}$ gene is highlighted. $\mathbf{g}$ Alignment of the AVRPM3 ${ }^{\mathrm{D} 3}$ protein sequences encoded by BgtE-20069b, and the duplicated BgtE-20069a paralog from Bgt_96224, and Bgt_94202. h Functional validation of AvrPm3d3 in transient assays in $N$. benthamiana. HR was scored 4-5 days after co-infiltration (Fusion-FX imager). All effector constructs were codon optimised for expression in $N$. benthamiana, synthesised without the signal peptide, and recombined into the plPKb004 expression vector. All Pm3 alleles were expressed as native wheat sequences in the pIPKb004 expression vector. Complete $N$. benthamiana leaf pictures are provided in a Source Data File. Results are consistent over at least two independent assays each consisting of 6-8 independent leaf replicates

NLR proteins. One hundred candidates from the effector benchmarking and all six candidates from the GWAS were codon optimised for expression in N. benthamiana (Supplementary Data 3), synthesised without the signal peptide, and screened for recognition by $P m 3 b, P m 3 c$ and $P m 3 d$ using our transient assays in $N$. benthamiana leaves (see Methods). We took advantage of the high sensitivity reading (HSR) technology embedded in the Fusion FX fluorescence imager (www.vilber.com) to quantitatively score the hypersensitive response 5 days after infiltration ${ }^{29,30}$. One $A v r$ candidate, BgtE-20002, commonly found by benchmarking and GWAS, induced strong HR when combined with $P m 3 b$ and weak HR with Pm3c, while the sequence variant BgtE-20002_I, induced strong HR both with $P m 3 b$ and $P m 3 c$ (Fig. 1d, Supplementary Fig. 5a, b). We observed no HR when both variants were coinfiltrated with $P m 3 a, P m 3 f, P m 3 d$ and $P m 3 e$, indicating that this effector is implicated in dual and specific recognition by the $P m 3 b$ and $P m 3 c$ alleles that have previously been shown to exhibit overlapping recognition spectra, with $P m 3 c$ representing the weaker allele ${ }^{35}$. Therefore, we concluded that BgtE-20002 is $A v r P m 3^{b 2 / c 2}$, the $A v r$ recognised by $P m 3 b$ and $P m 3 c$. Also, because $B g t E-20002$ is not polymorphic between the $P m 3 b / c$ avirulent isolate Bgt_96224 and the virulent isolate Bgt_94202, we conclude that $A v r P m 3^{b 2 / c 2}$ is genetically suppressed by the active $S v r P m 3^{a 1 / f 1}$ allele encoded in Bgt 94202, in a similar way as $A v r P m 3^{a 2 / f 228,32 \text {, }}$

A second Avr candidate, BgtE-20069b, only found in the benchmarking list, and encoded within a segmental duplication on chromosome 9 (Fig. 1e-g), induced HR in combination with Pm3d (Fig. 1h, Supplementary Fig. 5c). No HR was observed when BgtE-20069b was combined with Pm3a, Pm3f, Pm3b, Pm3c and $P m 3 e$, indicating that this effector gene is $A v r P m 3^{d 3}$, the $A v r$ recognised by $P m 3 d$. The $B g t E-20069 b$ copy corresponding to $A v r P m 3^{d 3}$, is deleted in the virulent isolate Bgt_94202. Its paralog, BgtE-20069a, has two non-synonymous SNPs compared to the $A v r$, and it is polymorphic between Bgt_96224, and Bgt_94202 (Fig. 1g). We used the same transient assays in N. benthamiana to test for possible recognition of both alleles of BgtE-20069a by $P m 3 d$, and we observed no HR (Fig. 1h). All together this data demonstrates that specificity of the AvrPm $3^{d 3}-P m 3 d$ interaction is based on specific recognition of one paralog of a duplicated effector gene, BgtE-20069b.

In a last series of functional validation assays, we attempted epitope tagging of AVRPM3 $3^{\mathrm{A} 2 / \mathrm{F} 2}$, $\mathrm{AVRPM}^{\mathrm{B} 2 / \mathrm{C} 2}$ and AVRPM3 ${ }^{\mathrm{D} 3}$. All effector proteins were $\mathrm{C}$ and $\mathrm{N}$ terminally fused to HA and FLAG epitope tags, and subsequently tested for (i) detectability on a western blot, and (ii) functionality in the N. benthamiana assay (Fig. 2, Supplementary Note 3). For AVRPM3 $3^{\mathrm{A} 2 / \mathrm{F} 2}$, $\mathrm{N}$ terminal $\mathrm{HA}$ and FLAG fusions were detectable but not the $\mathrm{C}$ terminally tagged constructs. We observed the opposite for AVRPM3 ${ }^{\mathrm{B} 2 / \mathrm{C} 2}$, where the $\mathrm{C}$ terminal $\mathrm{HA}$ and FLAG fusion were detectable but not the $\mathrm{N}$ terminal ones. For AVRPM $3^{\mathrm{D} 3}$, none of the epitope fusions was detectable on the western blot (Fig. 2a-c). We have also tested the functionality of the detectable HA-AVRPM3 $3^{\mathrm{A} 2 / \mathrm{F} 2}, \mathrm{AVRPM}_{3}{ }^{\mathrm{B} 2 /}$ $\mathrm{C} 2$-HA constructs in co-infiltration assays with their respective PM3 NLRs, and found that these epitope fusions did not interfere with AVR function (Fig. 2d-f, Supplementary Fig. 6). Together, these assays demonstrate that AVRPM3 protein function is sensitive to epitope fusions in a tag sequence and positiondependent manner.

AvrPm3 recognition is suppressed by Svr and NLR interactions. Genetic studies in F1 segregating populations of wheat powdery mildew have revealed that $\operatorname{SvP} P m 3^{a 1 / f 1}$ is genetically suppressing several Avrs of the Pm3 genes (including AvrPm $3^{b 2 / c 2}$ and $A v r P m 3^{d 3}$ ), but so far functional evidence was only provided for $A v r P m 3^{a 2 / f 228}$. Here, with the cloning of two novel AvrPm3 genes, we had a unique opportunity to functionally validate that SvrPm3 $3^{a 1 / f 1}$ can act as a suppressor of $A v r$ recognition for additional (probably all) AvrPm3 genes ${ }^{28}$. We co-expressed $A v r P m 3^{b 2 / c 2}$, and $A v r P m 3^{d 3}$ with $P m 3 b$ and $P m 3 d$, respectively, in presence of the active $S v r P m 3^{a 1 / f 1}$ allele originating from the mildew isolate Bgt_94202 28 . HR was assessed relative to a control where the active $S v r P m 3^{a 1 / f 1}$ suppressor allele was replaced by the inactive $s v r P m 3^{a 1 / f 1}$ allele encoded by the mildew isolate Bgt_96224, as previously described ${ }^{28}$. As an additional control, we also assayed suppression of the $A v r P m 3^{a 2 / f 2}-P m 3 a$ interaction using the same experimental set-up (see Methods). As previously demonstrated $^{28}, A v r P m 3^{22 / f 2}$ recognition was suppressed by $\operatorname{SvrPm} 3^{a 1 / f 1}$ and resulted in significant reduction of HR intensity in our control assay (Fig. 3a), thus demonstrating the functionality of our improved experimental system based on codonoptimised constructs and HSR imaging. Consistent with previous genetic evidence from Bourras and colleagues ${ }^{28}$, we found in two independent assays that recognition of $A v r P m 3^{b 2 / c 2}$ and $A v r P m 3^{d 3}$ was also suppressed by $\operatorname{SvP} P m 3^{a 1 / f 1}$ and resulted in significant reduction of HR intensity (Student $t$-test, $p$-value $<$ 0.05) (Fig. 3b, c). We also produced $\mathrm{C}$ and $\mathrm{N}$ terminal HA and FLAG epitope tag fusions of the active SVRPM3 $3^{\mathrm{A} 1 / \mathrm{F} 1}$ variant and found that all constructs were detectable on the western blot, suggesting significant differences between AVRPM3 and SVRPM3 proteins in terms of tolerance to epitope fusions (Fig. 3d). We have therefore tagged the inactive svrPM3 ${ }^{\mathrm{Al} / \mathrm{F} 1}$ variant that we use as negative control for AVRPM3 suppression, and tested for protein expression levels as compared to the active suppressor. We found no difference in protein abundance 
a

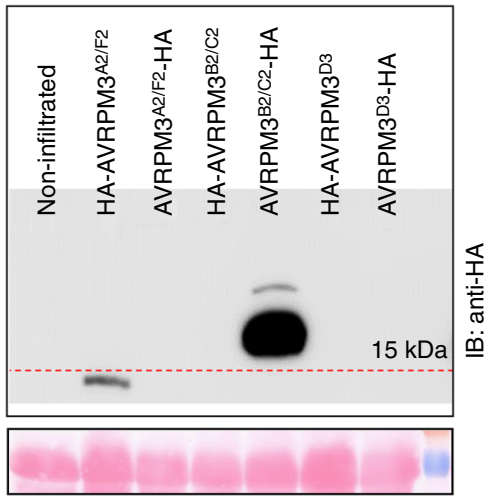

b
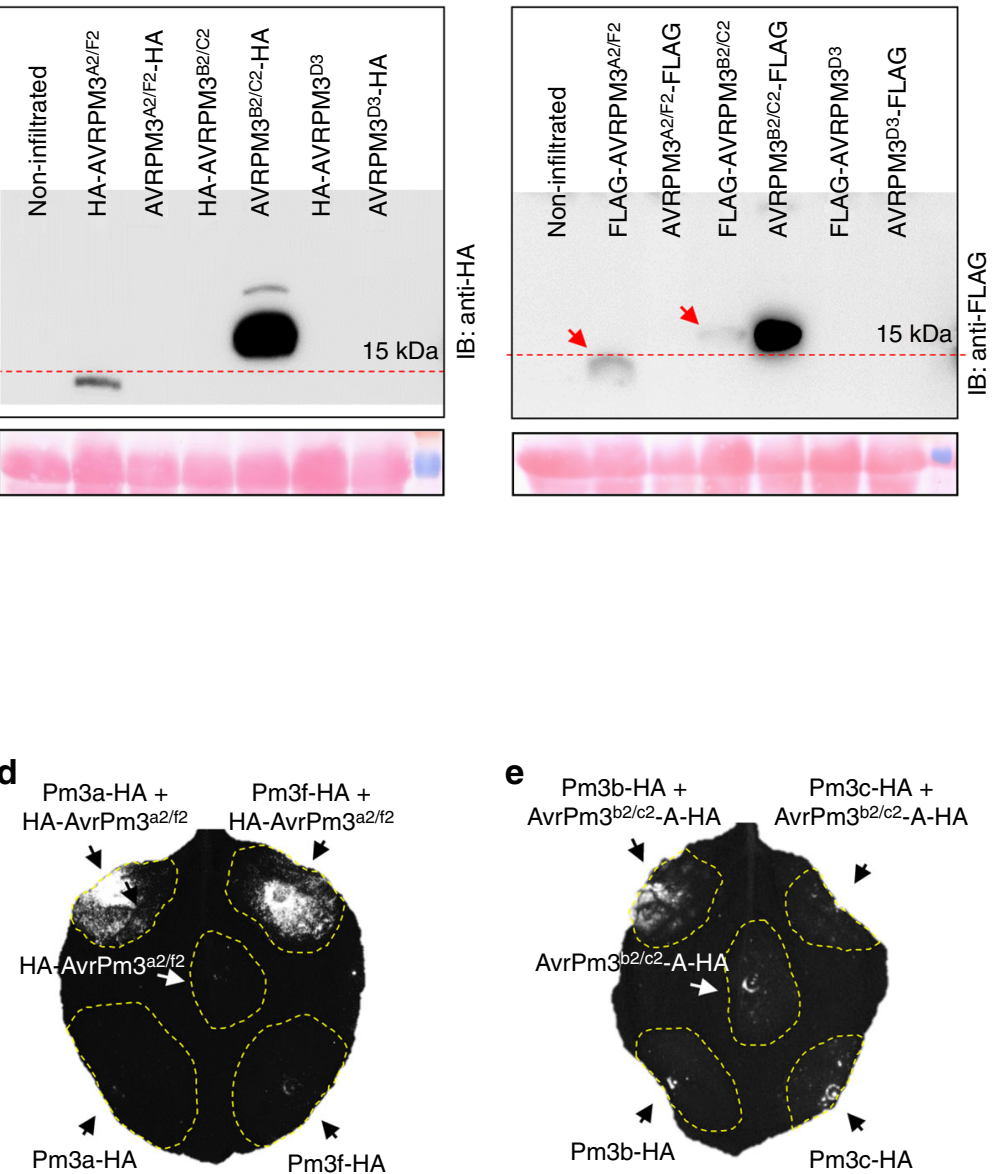

e

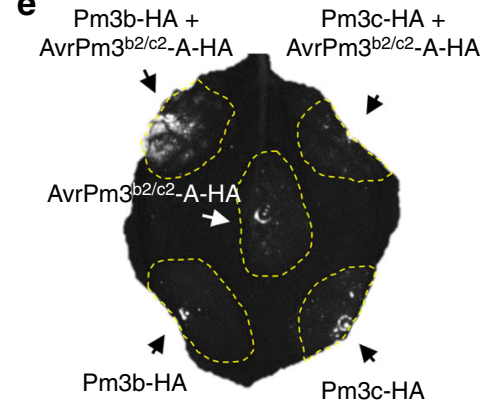

C

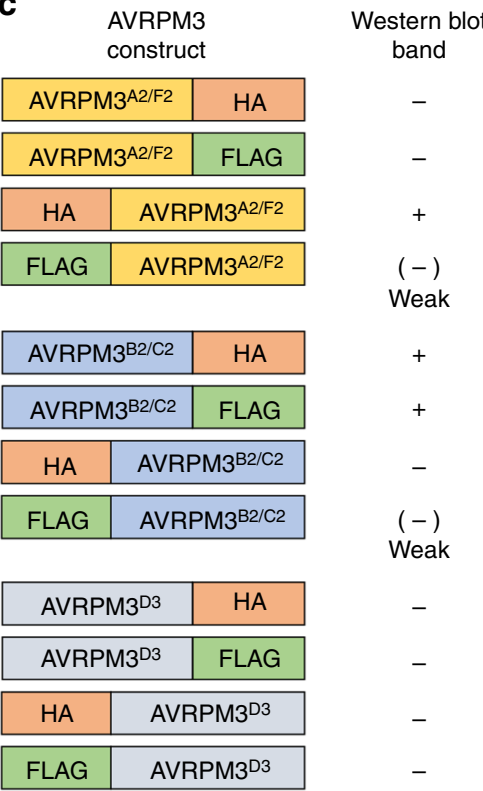

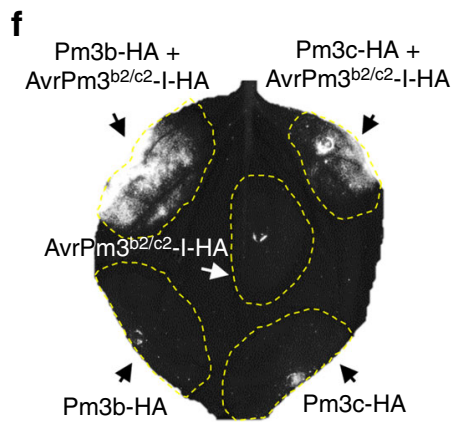

Fig. 2 Impact of epitope fusion of the AVRPM3 protein expression and detectability. a, b Western blot detection of $\mathrm{C}$ and $\mathrm{N}$ terminal HA (a) and FLAG (b) epitope tag fusion of AVRPM3A2/F2, AVRPM3 ${ }^{B 2 / C 2}$ and AVRPM3 ${ }^{D 3}$ (upper panel) and Ponceau staining of the Western blot membrane (lower panel) are depicted. Uncropped western blot images are provided in a Source Data File. c Graphical summary of AVRPM3 protein tagability based on detection of the protein on a western blot, demonstrating significantly different impacts of epitope fusions based on tag position and sequence. $\mathbf{d}-\mathbf{f}$ experimental assessment of HA tagged AVR variants in functional validation assays in N. benthamiana. Protein expression data is provided in Supplementary Fig. 6. HA$A V R P M 3^{A 2} / F 2$ (d), AVRPM3 ${ }^{B 2} / C_{-}-A-H A(e)$ and AVRPM3 B2/C2-I-HA (f), co-expressed with their respective NLRs. HR was assessed using HSR imaging 4-5 days after Agrobacterium infiltration. Results are consistent over at least two independent assays each consisting of 6-8 independent leaf replicates

between HA-SVRPM3 ${ }^{\mathrm{A1} / \mathrm{F} 1}$, and HA-svrPM3 ${ }^{\mathrm{A1} / \mathrm{F} 1}$ (Fig. 3e), and we could show that the tag is also not interfering with SVRPM3 ${ }^{\mathrm{A} 1 / \mathrm{F} 1}$ function (Fig. $3 \mathrm{f}, \mathrm{g}$ ). These data demonstrate that differences between active and inactive suppressor variants are not due to differences in protein expression. Based on this data, we could confidently test the hypothesis that $\operatorname{SvP} P m 3^{a 1 / f 1}$ is possibly acting as a transcriptional or translational suppressor of AvrPm3 or $P m 3$, based on a previous observation suggesting this effector encodes a fungal ribonuclease-like protein ${ }^{32}$. To do so, we tested protein expression levels of AVRPM3 ${ }^{\mathrm{A} 2 / \mathrm{F} 2}$, $A V R P M 3^{B 2 / C 2}$ and individual PM3 NLRs in presence vs. absence of SVRPM3 $3^{\mathrm{A} 1 / \mathrm{F} 1}$ and found no differences (Supplementary Fig. 7). These results demonstrate that $S v r P m 3^{a 1 / f 1}$ can indeed act as a suppressor of recognition of several $A v r P m 3$ genes, and suggest this function is not based on an AvrPm3 or Pm3-specific ribonuclease activity but occurs at the level of protein-protein interactions.

In addition to gene suppression based on the action of SvrPm $3^{a 1 / f 1}$, evidence from genetic studies in wheat and transient assays with auto-activated PM3 constructs ${ }^{26,28}$, indicated that the AvrPm3-Pm3 interactions can also be suppressed by inter-allelic interactions between the PM3 NLRs.
Here, with the cloning of additional AvrPm3 genes and the availability of an improved cell death assay in N. benthamiana, we had a unique opportunity to test for inter-allelic NLR-NLR interactions in presence of the AVR proteins. We assayed the suppression activity of $P m 3 a-f$ and the non-functional ancestral allele $P m 3 C S$ in transient co-expression assays when each allele was combined with $A v r P m 3^{a 2 / f 2}-P m 3 a, A v r P m 3^{a 2 / f 2}-P m 3 f$, $A v r P m 3^{b 2 / c 2}-\mathrm{I}-P m 3 b, A v r P m 3^{b 2 / c 2}-\mathrm{I}-\mathrm{Pm} 3 c$, and $A v r P m 3^{d 3}-\mathrm{Pm} 3 d$ (Supplementary Figs. 8 and 9, Supplementary Note 4). We found that $P m 3 a$ and $P m 3 f$ had no NLR suppression activity and were mostly suppressed by the other $P m 3$ alleles. $P m 3 e$ was only active on the weaker $P m 3 c$ and $P m 3 f$ alleles, while $P m 3 b$, $P m 3 c$ and $P m 3 d$ had reciprocal suppression capacity and acted as the strongest suppressors among the functional Pm3 NLRs. Here we conclude that inter-allelic suppression occurs upon AVR-dependent activation of the PM3 NLRs, and it is NLR sequence dependent.

The AVRPM3 proteins belong to a group of related effectors. $A v r P m 3^{b 2 / c 2}$ and $A v r P m 3^{d 3}$ are not members of the $A v r P m 3^{a 2 / f 2}$ effector gene family E008 but belong to the mildew effector 
a
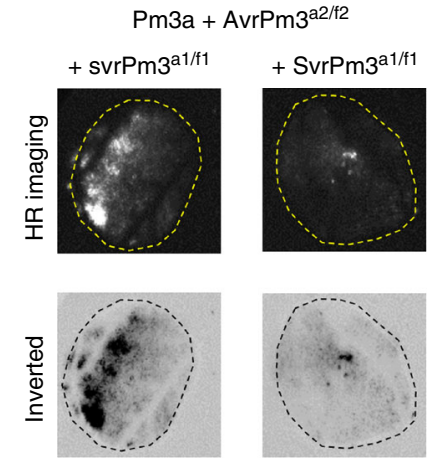

$\mathrm{Pm} 3 \mathrm{a}+\mathrm{AvrPm} 3^{\mathrm{a} / \mathrm{f} 2}$

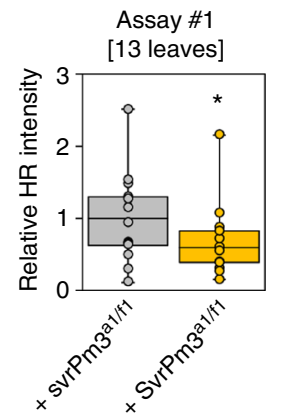

b
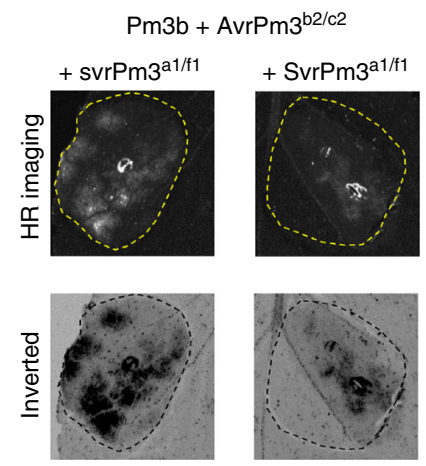

$\mathrm{Pm} 3 \mathrm{~b}+\mathrm{AvrPm} 3^{\mathrm{b} 2 / \mathrm{c} 2}$

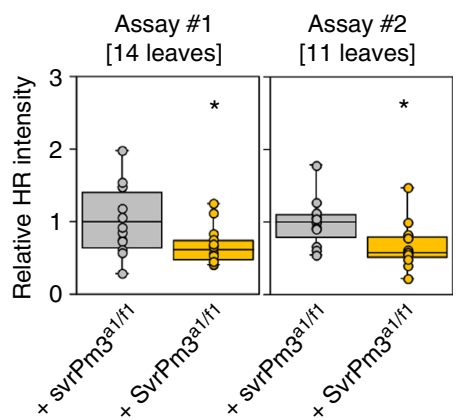

f

$\mathrm{AvrPm}^{\mathrm{a} 2 / \mathrm{f} 2}+\mathrm{AvrPm}^{\mathrm{a} / \mathrm{f} 2}+$ $\mathrm{Pm} 3 f^{\mathrm{L} 456 \mathrm{P} / \mathrm{Y} 458 \mathrm{H}} \quad \mathrm{Pm} \mathrm{f}^{\mathrm{L} 456 \mathrm{P} / \mathrm{Y} 458 \mathrm{H}}$ + svrPm3 $^{\mathrm{a} 1 / \mathrm{f1}}+\mathrm{SvrPm}^{\mathrm{a} 1 / \mathrm{f1}}$

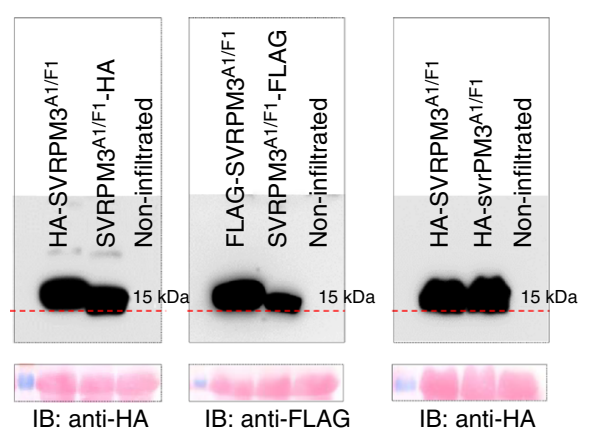

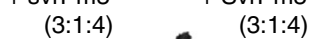
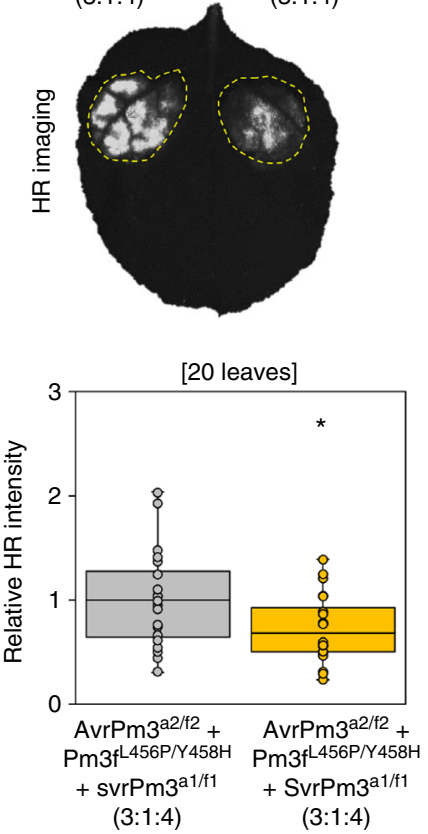

c
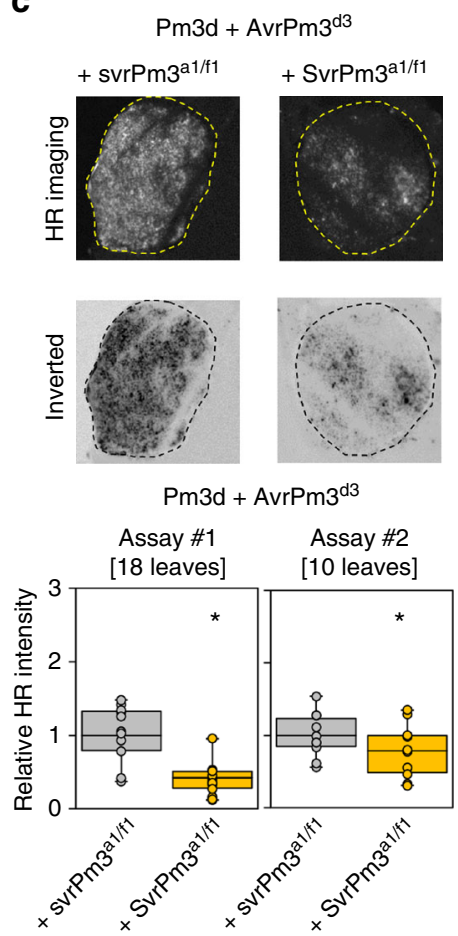
g

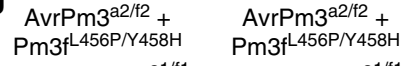 + HA-svrPm3 $3^{\mathrm{a} 1 / \mathrm{f} 1}+\mathrm{HA}-\mathrm{SvrPm} \mathrm{a}^{\mathrm{a} 1 / \mathrm{f} 1}$
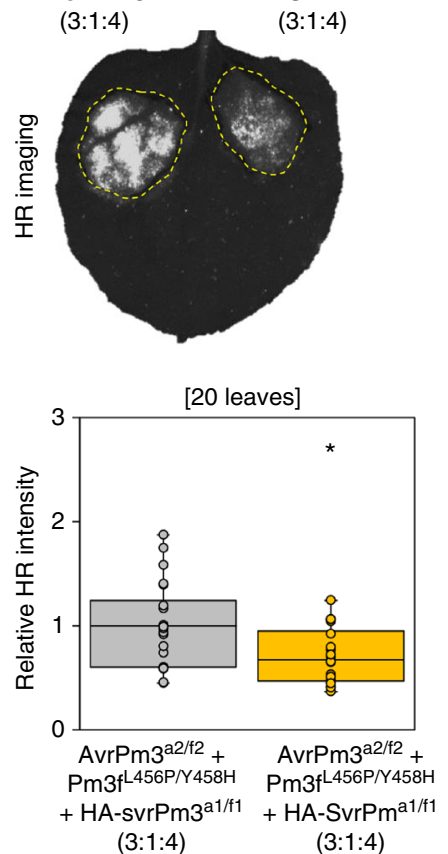

families E018 and E034, respectively ${ }^{34}$ (Supplementary Figs. 3 and 10). Sequence alignment of the AVRPM3 $3^{\mathrm{A} 2 / \mathrm{F} 2}, \mathrm{AVRPM}^{\mathrm{B} 2 / \mathrm{C} 2}$, and $A V R P M 33^{D 3}$ full proteins showed that the three AVRs share low identity (14.5 to $19.9 \%$, Supplementary Fig. 11). Sequence alignment of the three effector families revealed conservation of the signal peptide, a conserved Hydrophobic x C $(\mathrm{HxC})$ motif, and a characteristic conserved pattern of alternating hydrophobic residues, (Supplementary Fig. 12). Analysis of the phylogenetic relationships between the AVRPM3 families suggests that $\mathrm{AVRPM}^{\mathrm{A} 2 / \mathrm{F} 2}$ and AVRPM $3^{\mathrm{B} 2 / \mathrm{C} 2}$ belong to the same superfamily which groups separately from AVRPM3 $3^{\mathrm{D}}$, and all three families are phylogenetically distinct from the SVRPM $3^{\mathrm{A} 1 / \mathrm{F} 1}$ family (Supplementary Fig. 13).

The PM3 NLRs are highly similar on the protein level with only few amino acid differences ${ }^{24}$. We therefore hypothesised that a basis for specificity could be that the sequence unrelated AVRPM3 effectors are encoding for structurally similar proteins, based on conservation of amino-acid properties and the position of structural residues such as cysteines and prolines (Supplementary Fig. 12). We applied AVRPM3 $3^{\mathrm{A} 2 / \mathrm{F} 2}, \mathrm{AVRPM}^{\mathrm{B} 2 / \mathrm{C} 2}$, and 
Fig. 3 Suppression of AvrPm3-Pm3 recognition by SvrPm3 ${ }^{a 1 / f 1}$. a-c Suppression of the recognition of $A v r P m 3^{a 2 / f 2}$ (a), AvrPm3 $3^{b 2 / c 2}$ (b) and $A v r P m 3^{d 3}$ (c) in

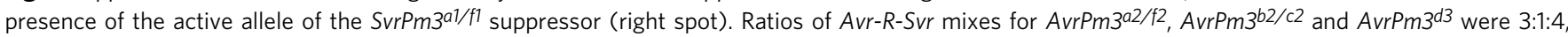
4:1:4, and 2:1:4 respectively. HR is compared to a negative control in presence of the inactive suppressor allele $s v r P m 3^{a 1 / f 1}$ (left spot). Results are consistent over at least two independent assays each consisting of at least 10 independent leaf replicates. HR quantification in presence of the inactive vs. active suppressor is depicted in the lower panels. Number of independent leaf replicates is indicated. Complete $N$. benthamiana leaf pictures are provided in Source Data File. $\mathbf{d}$ Western blot detection of $C$ and $N$ terminal HA and FLAG epitope fusions of the active SVRPM $3^{A 1 / F 1}$ and Ponceau staining of the membrane (lower panel) are depicted. e Western blot detection HA-SVRPM3A1/F1 and HA-svrPM3A1/F1 suppressors demonstrating there is no difference in protein abundance between active vs. inactive alleles, respectively. Uncropped Western blot images are provided in Source Data File. $\mathbf{f}, \mathbf{g}$ Functionality of

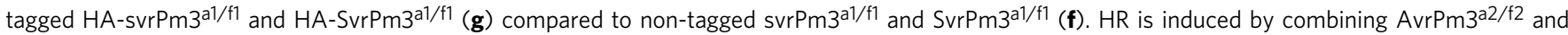
Pm3f $L 456 \mathrm{P} / \mathrm{Y} 458 \mathrm{H}$. Suppression is assessed by comparing active vs. inactive suppressors, as originally described in Bourras et al. ${ }^{28}$. Avr- $R$-Svr ratios are indicated. HR was measured using HSR imaging 3 days post $N$. benthamiana agro-infiltration ( $f-g$, upper panel). HR Quantification from the same assays revealed by HSR imaging ( $f-g$ lower panels). In both cases, the presence of the active suppressor allele (SvrPm3 $3^{\text {al/f1 }}$ ) resulted in significant reduction of $H R$ as compared to the inactive alleles (svrPm3 $3^{\mathrm{a} 1 / \mathrm{f1}}$ ) independently of the epitope, demonstrating that HA-svrPm3 $3^{\mathrm{a} 1 / \mathrm{f1}}$ and $\mathrm{HA}-\mathrm{SvrPm} 3^{\mathrm{a} 1 / \mathrm{f1}}$ are functional. Number of independent leaf replicates is indicated. Statistical significance in a-c, $\mathrm{f}, \mathrm{g}$ was assessed with a two-sided Student $\mathrm{t}$-Test for paired data and indicated with $\left({ }^{*} ; p<0.05\right)$. Mean values are indicated by the middle line in the boxplot. Individual data points are plotted along the whiskers delineating minimum and maximum values. Raw data underlying the reported averages in a-c, $f, g$ are provided in a Source Data File

AVRPM3 ${ }^{\mathrm{D} 3}$ to secondary structure modelling using four different prediction algorithms available at the Quick2D platform (https:// toolkit.tuebingen.mpg.de/\#/tools/quick $2 \mathrm{~d}^{36}$,). All modelling methods have consistently predicted the presence of one alpha helix followed by three to four beta-sheets for all three AVRs (Supplementary Fig. 14a). To further substantiate these results, we applied the AVRPM3 ${ }^{\mathrm{A} 2 / \mathrm{F} 2}$, AVRPM3 $3^{\mathrm{B} 2 / \mathrm{C} 2}$, and AVRPM3 ${ }^{\mathrm{D} 3}$ effector family members to three dimensional structural modelling using the RaptorX platform (raptorx.uchicago.edu ${ }^{37}$,). We found no statistically robust structural model common to all three families or to all members of the same family (Supplementary Data 5). However, despite differences in the templates assigned by RaptorX to individual members, we found that several of the tertiary folds predicted for the AVRPM3 ${ }^{\mathrm{A} 2 / \mathrm{F} 2}$ and AVRPM3 ${ }^{\mathrm{B} 2 / \mathrm{C} 2}$ families consisted of one central helix facing three to four betasheets, reminiscent of the previously predicted secondary folds (Supplementary Fig. 14b, c). In particular, this putative tertiary structure was also predicted for the AVRPM3 ${ }^{\mathrm{A} 2 / \mathrm{F} 2}$ and $\mathrm{AVRPM}^{\mathrm{B} 2 / \mathrm{C} 2}$ avirulence proteins. Therefore, based on protein alignments, phylogeny, and in silico secondary and tertiary structure modelling, we suggest that AVRPM3 ${ }^{\mathrm{A} 2 / \mathrm{F} 2}$ and AVRPM $3^{\text {B2/C2 }}$ are possibly encoding for structurally similar proteins. Finally, all AvrPm3 genes are highly induced upon infection, and all encode for typical pathogen effector proteins (Supplementary Note 5, Supplementary Fig. 15). We therefore propose that the AvrPm3 genes are possibly acting as bona-fide virulence factors suppressing host immunity.

$A v r P m 3^{b 2 / c 2}$ and $A v r P m 3^{d 3}$ conservation in mildew populations. In a previous study, McNally and colleagues ${ }^{29}$ found limited sequence diversity for $A v r P m 3^{a 2 / f 2}$ in worldwide mildew populations, and a high frequency of the recognised haplotype variant. These observations are contrasting with the general hypothesis arguing that avirulence genes are highly polymorphic in pathogen populations, and that active variants tend to be lost because of selective pressure to escape recognition from the host immune system ${ }^{29}$. To study the genetic diversity of $A v r P m 3^{b 2 / c 2}$ and $A v r P m 3^{d 3}$, we applied a collection of 185 powdery mildew isolates with a worldwide geographical distribution to haplotype mining as previously described by McNally and colleagues ${ }^{29}$. The full sequences of $A v r P m 3^{b 2 / c 2}$ and $A v r P m 3^{d 3}$ were recovered using genome sequencing data or PCR gene amplification and subsequent sequencing (see Methods). For $A v r P m 3^{b 2 / c 2}$, we identified 10 non-synonymous mutations across the gene, including 8 in the mature protein resulting in 7 protein variants (Fig. 4a, b, Supplementary Fig. 16). Based on those haplotype sequences, we found a $\mathrm{pN} / \mathrm{pS}$ (ratio of polymorphic nonsynonymous $(\mathrm{pN})$ to polymorphic synonymous ( $\mathrm{pS}$ ) sites within a species) of 1.26 indicating that the $A v r P m 3^{b 2 / c 2}$ gene is under diversifying selection, which is consistent with previous finding by Müller et al. ${ }^{34}$ and McNally et al. ${ }^{29}$ showing that the whole $A v r P m 3^{b 2 / c 2}$ and $A v r P m 3^{a 2 / f 2}$ families are under diversifying selection. All haplotypes of $A v r P m 3^{b 2 / c 2}$ were codon optimised for expression in $N$. benthamiana, synthesised without the signal peptide and screened for recognition by $P m 3 b$ and $P m 3 c$. No haplotype besides the previously validated $A v r P m 3^{b 2 / c 2}-\mathrm{A}$ variant from Bgt_96224 and the AvrPm3 $3^{b 2 / c 2}$-I from Bgt_07237, induced HR when combined with $P m 3 b$ and $P m 3 c$ in $N$. benthamiana (Fig. 4b). Protein expression assays demonstrated that loss of $\mathrm{AVRPM} 3^{\mathrm{B} 2 / \mathrm{C} 2}$ recognition is not due to absence of the protein as all haplovariants are equally detectable on a western blot (Fig. 4c, Supplementary Fig. 17). We found that the AvrPm3 $3^{b 2 / c 2}$-I is only present in the Swiss population and represented by only one isolate. Interestingly, the $A v r P m 3^{b 2 / c 2}-\mathrm{A}$ variant was the most frequent haplotype found in all subpopulations, and in two regions, USA and Israel, this active $A v r$ variant was more frequent than the active $A v r P m 3^{a 2 / f 2}-\mathrm{A}$ (Fig. 4a). For $A v r P m 3^{d 3}$, we identified 17 non-synonymous mutations across the whole gene, including 15 in the mature protein. However, it was not possible to distinguish between the SNPs that should be assigned to a haplotype of the BgtE-20069a or the BgtE-20069b paralogs (i.e. AvrPm3 $3^{d 3}$ ) which only differ by two residues (Fig. 1g). Therefore, while we could not resolve the haplotype diversity of $A v r P m 3^{d 3}$ which shows high level of copy number variation in mildew isolates $\left(A v r P m 3^{b 2 / c 2}\right.$ is present in a single copy only, see Supplementary Fig. 18), these results show that at least one paralog of the $A v r P m 3^{d 3}$ effector can be found globally in all isolates. Here we conclude that data from diversifying selection studies and global population genetics provide further evidence substantiating that the AvrPm3 effectors are important virulence factors.

We also assessed the individual impact on AVR recognition of the 7 amino-acid variations in the mature protein of AVRPM3 ${ }^{B 2 /}$ $\mathrm{C} 2$ found in our natural diversity (residue changes in the signal peptide were not tested) (Fig. 4d). For AVRPM3 ${ }^{D 3}, 14$ aminoacid variations were similarly assessed which very likely include polymorphic residues from its paralog BgtE-20069a, because these could not be distinguished in our haplotype mining (Fig. 4e). Every single amino acid variation was used to generate point mutants in AVRPM $3^{\mathrm{B} 2 / \mathrm{C} 2}$ and $\mathrm{AVRPM} 3^{\mathrm{D} 3}$, and all were screened for recognition by their respective NLRs using transient assays in $N$. benthamiana as previously described ${ }^{29}$. We found that most of the single amino acid mutations disrupted the recognition of $A V R P M 3^{B 2 / C 2}$ and $A V R P M 3^{D} 3$ by their respective 
a

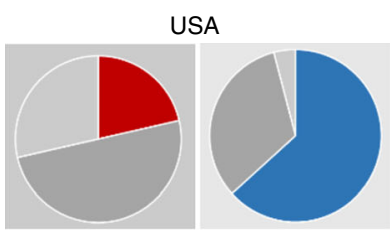

$\operatorname{AvrPm} 3^{\mathrm{a} 2 / f 2}$

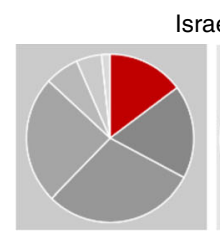

AvrPm3 $3^{a / f 2}$ srael

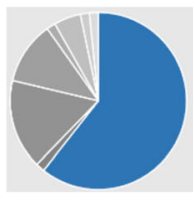

AvrPm3 $3^{\mathrm{b} 2 / \mathrm{c} 2}$

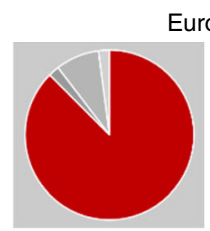

AvrPm3a2/f2
Europe

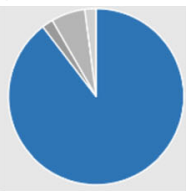

$\operatorname{AvrPm} 3^{b 2 / c 2}$

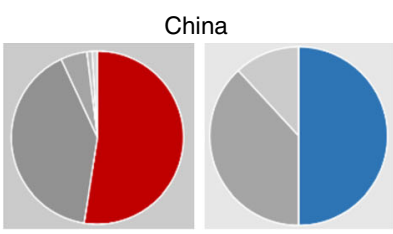

AvrPm3 $3^{a / f 2}$

$\operatorname{AvrPm}^{\mathrm{b} 2 / c 2}$
Active AvrPm3 $3^{a 2 / t 2}$ variants

b

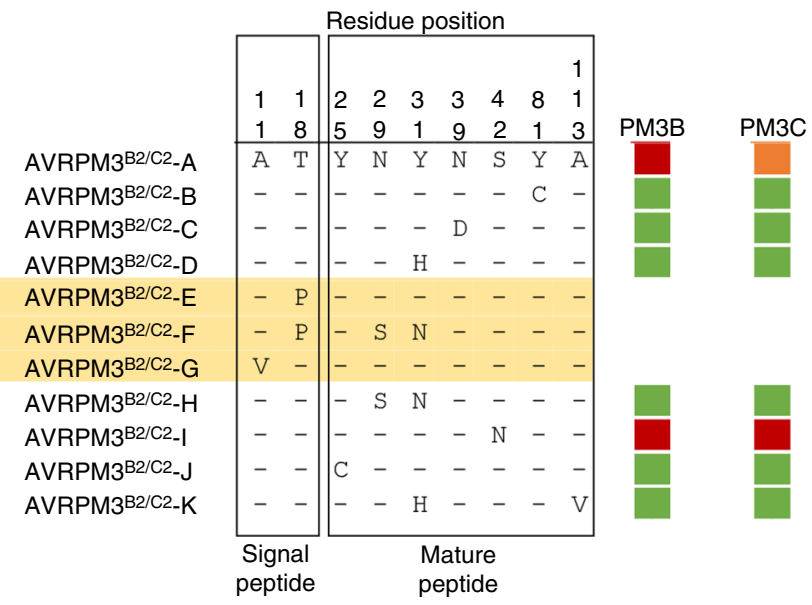

d

$>$ AVRPM $3^{\mathrm{B} 2 / \mathrm{C} 2}$

$\begin{array}{llllll}1 & 10 & 20 & 30 & 40 & 50\end{array}$

MKISALESVAALLSQSMTVLGYLFYRCGNDY ITERALIN I ISMEHKKLTG

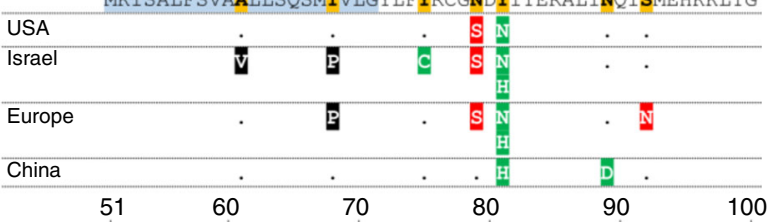

QGSSADSFPGGRATAEVTFWEPSISNPGTYLDIKVKFDIYRQMLSFEVSS

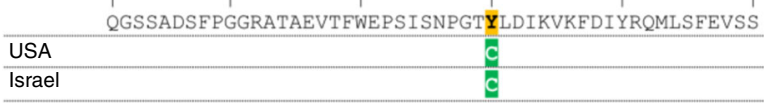

Europe

China

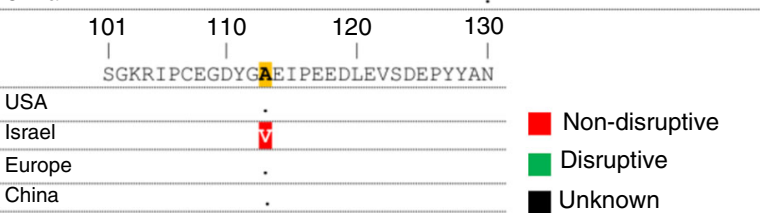

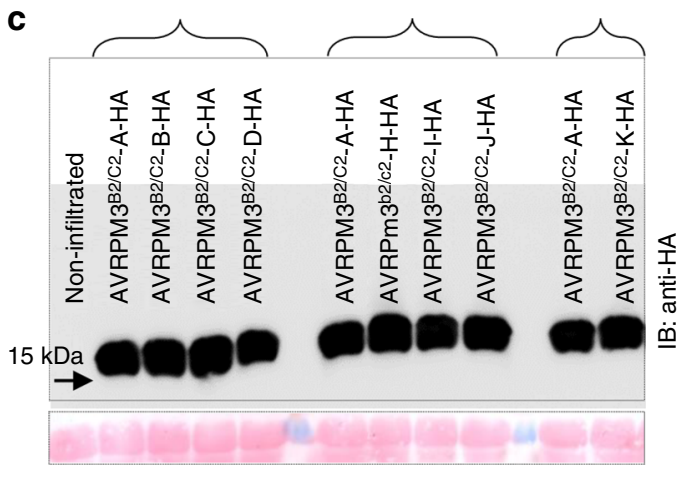

$\mathbf{e}$

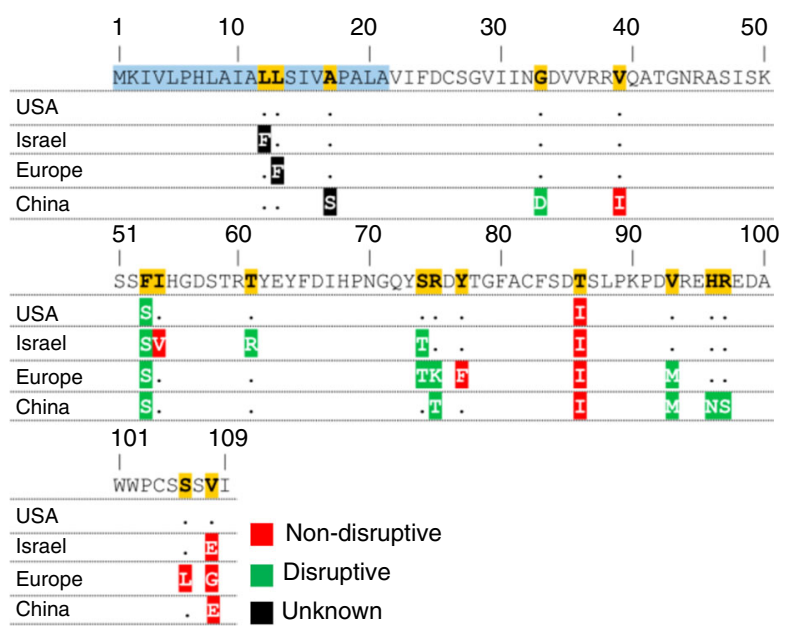

Fig. 4 Functional characterisation of $A V R P M 3^{B 2 / C 2}$ and $A V R P M 3^{D 3}$ based on natural and artificial sequence variation. a Geographical distribution and frequency of $A v r P m 3^{b 2 / c 2}$ sequence haplotypes encoding active AVRPM3B2/C2 virulence protein variant as compared to AvrPm3a2/f2. Underlying source

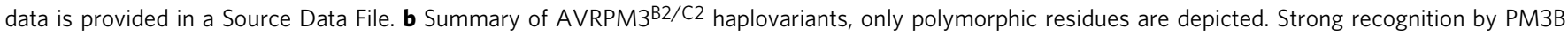
and PM3C based on $N$. benthamiana infiltration assays are indicated with a red box, and weak recognition with an orange box. All assays are based on expression of the mature protein after removal of the signal peptide. Therefore, haplovariants $E$ and $G$, and haplovariant $F$ were not tested because the mature protein sequence is identical to variant $A$ and variant $H$, respectively. $\mathbf{c}$ Western blot detection (upper panel) of $C$ terminal $H A$ epitope fusion to the mature proteins of the $A V R P M 3^{B 2 / C 2}$ haplovariants. Ponceau staining of the western blot membranes is depicted in the lower panel. Braces indicate samples where all constructs were combined on the same leaf and rotated together with AVRPM3 ${ }^{B 2 / C 2}$-A as a reference. Uncropped Western blot images are provided in a Source Data File. d, e Functional characterisation of the impact of natural sequence diversity of AVRPM3 ${ }^{B 2} / C 2$ (d) and AVRPM 3 D3 (e) on AVR function

NLRs (Fig. 4d, e), suggesting that selective pressure to escape $R$ gene recognition is a driving force for sequence diversification of $\mathrm{AVRPM}^{\mathrm{B} 2 / \mathrm{C} 2}$ and AVRPM3 ${ }^{\mathrm{D} 3}$. Protein expression analysis demonstrated that loss of $\mathrm{AVRPM} 3^{\mathrm{B} 2 / \mathrm{C} 2}$ recognition is not due to absence of the protein as all point mutants are equally detectable on a western blot (Supplementary Fig. 19). Interestingly, we found that most of the polymorphic positions in AVRPM $3^{\mathrm{B} 2 / \mathrm{C} 2}$, including 3 out of the 4 disruptive mutations, are located towards the N-terminal end within a stretch of 18 residues (aa 25-42), immediately after the predicted signal 
a

$>\mathrm{AVRPM}^{\mathrm{B} 2 / \mathrm{C} 2}$ mature protein

$>$ Bgt-51460 mature protein

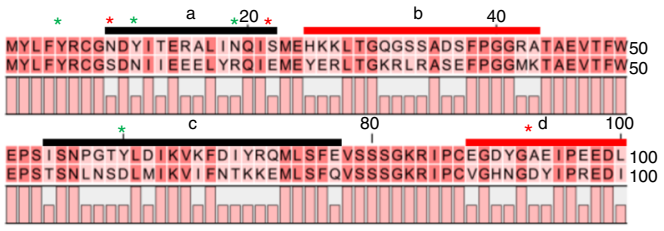

EVSDEPYYAN 110
QVPDNDMYDD 110

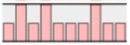

C

$\mathrm{AvrPm}^{\mathrm{b} 2 / \mathrm{c} 2} \# 6 \quad \mathrm{AvrPm}^{\mathrm{b} 2 / \mathrm{c} 2}$ $+\mathrm{Pm} 3 \mathrm{c}$

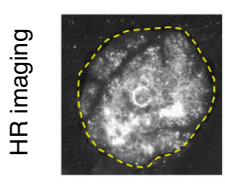

$+\mathrm{Pm} 3 \mathrm{c}$

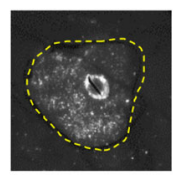

$\mathrm{AvrPm}^{\mathrm{b} 2 / \mathrm{c} 2} \# 6$ $+\mathrm{Pm} 3 \mathrm{~b}$

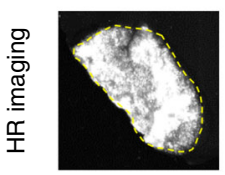

$\mathrm{AvrPm}^{\mathrm{b} 2 / \mathrm{c} 2}$

$+\mathrm{Pm} 3 \mathrm{~b}$
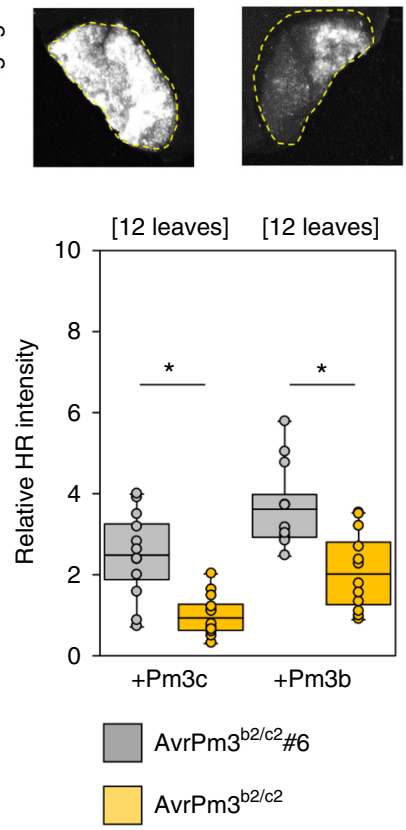

d b

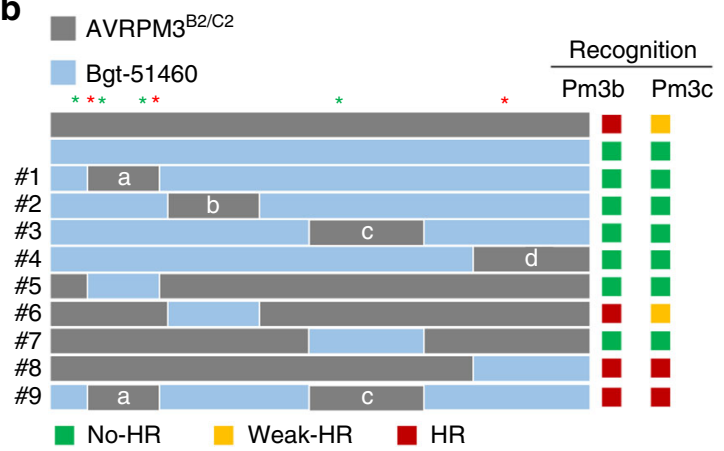

$\mathrm{AvrPm}^{\mathrm{b} 2 / \mathrm{c} 2} \# 8 \quad \mathrm{AvrPm}^{\mathrm{b} 2 / \mathrm{c} 2}$ $+\mathrm{Pm} 3 \mathrm{c}$

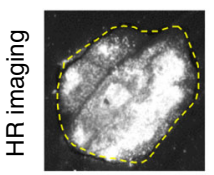

$+\mathrm{Pm} 3 \mathrm{c}$

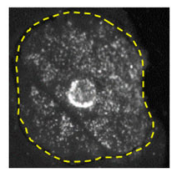

$\mathrm{AvrPm}^{\mathrm{b} 2 / \mathrm{c} 2} \# 8$

$+\mathrm{Pm} 3 \mathrm{~b}$

$\mathrm{AvrPm}^{\mathrm{b} 2 / \mathrm{c} 2}$

$+\mathrm{Pm} 3 \mathrm{~b}$
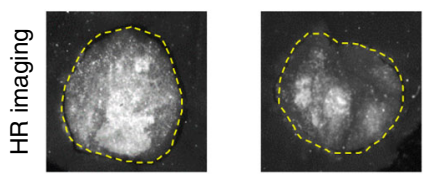

e

e $\quad \mathrm{AvrPm}^{\mathrm{b} 2 / \mathrm{c} 2} \# 9 \quad \quad \mathrm{AvrPm}^{\mathrm{b} 2 / \mathrm{c} 2}$

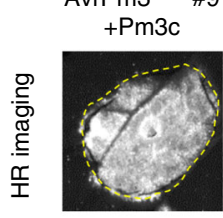

$+\mathrm{Pm} 3 \mathrm{c}$

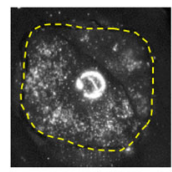

$\mathrm{AvrPm}^{\mathrm{b} 2 / \mathrm{c} 2} \# 9$ $+\mathrm{Pm} 3 \mathrm{~b}$

$\mathrm{AvrPm}^{\mathrm{b} 2 / \mathrm{c} 2}$

$+\mathrm{Pm} 3 \mathrm{~b}$
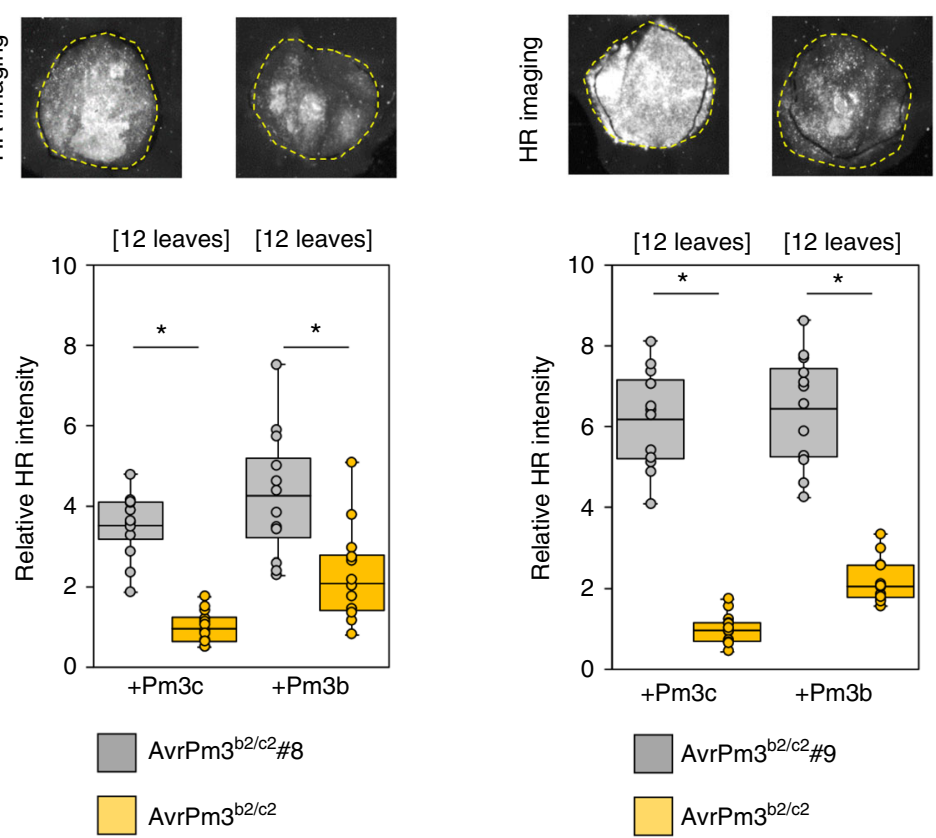

Fig. 5 Consequence of synthetic domain swaps on the recognition of $A V R P M 3^{B 2} / C 2$. $\mathbf{a}-\mathbf{b}$ Consequence of synthetic domain swaps on the $A V R P M 3^{B 2 / C 2} A V R$ function. a Protein sequence alignment of the mature protein of AVRPM3 ${ }^{B} / \mathrm{C2}_{-} \mathrm{A}$ and the closest family member Bgt-51460. Swapped domains are indicated and labelled a, b, c and d. Domains that do not abolish AVR recognition when introduced from Bgt-51460 into AVRPM3 ${ }^{B 2 / C 2}$ are indicated in red. $\mathbf{b}$ Schematic representation of the protein domains swapped between AVRPM3B2/C2 (grey) and Bgt-51460 (blue). $\mathbf{a}$, b Position of the residues identified from the natural sequence diversity (Fig. 4b, d) are indicated with asterisks. The impact of individual residues on AVR recognition is indicated with green for mutations with a disruptive effect, and red for mutations, with a neutral effect, according to the results summarised in Fig. 4b, d. c-e Transient expression assays in $\mathrm{N}$. benthamiana indicating recognition (upper panel) and HR quantification (lower panel) for AVRPM3 ${ }^{B 2 / C 2}$ swaps \#6 (c), swap \#8 (d) and swap \#9 (e) by $P m 3 c$, and Pm3b. The number or independent leaf replicates is indicated. Mean values are indicated by the middle line in the boxplot. Individual data points are plotted along the whiskers delineating minimum and maximum values. Statistical significance was assessed with a two-sided Student's $t$-test for paired data and indicated with $\left({ }^{*} p<0.05\right)$. Complete $N$. benthamiana leaf pictures and raw data underlying the reported averages are provided in a Source Data File

peptide cleavage site (Fig. 4d). By contrast, most of the natural diversity in AVRPM3 ${ }^{\mathrm{D} 3}$ and its duplication BgtE-20069a, including 5 out of the 8 residues disrupting recognition, is found within a stretch of 24 residues (aa 74-97) towards the C-terminal end of the protein (Fig. 4e). This data suggests that specific domains of the AVR proteins are involved in recognition, and these seem to be located in distinctly different regions of AVRPM $^{\mathrm{B} 2 / \mathrm{C} 2}$ and AVRPM3 ${ }^{\mathrm{D} 3}$.
We therefore designed domain swaps covering different stretches of residues polymorphic between AVRPM $3^{\mathrm{B} 2 / \mathrm{C} 2}$ and $\mathrm{AVRPM}^{\mathrm{D} 3}$ and the closest members of their effector families, BGT-51460 and BGTE-5883, respectively (Fig. 5a, b, Supplementary Fig. 20a, b, Supplementary Note 6). For both AVR proteins we found that NLR-recognition is dependent on multiple distinct protein regions that overlap with regions with higher level of polymorphism in worldwide mildew accessions (Fig. 5b, 
Supplementary Fig. 20b). For AVRPM3 ${ }^{\mathrm{D} 3}$, all single region exchanges resulted in loss of AVR recognition, except for one highly conservative swap (construct \#6) where only a few residues at the $\mathrm{C}$ and $\mathrm{N}$ termini were exchanged with the corresponding sequence from BGTE-5883 (Supplementary Fig. 20b, c). For AVRPM $3^{\mathrm{B} 2 / \mathrm{C} 2}$, protein expression assay indicated all chimeric proteins can be detected on a western blot thus demonstrating that loss of recognition in our assays is not based on loss of AVR protein (Supplementary Fig. 21). We found that segments b and $d$ from AVRPM $3^{\mathrm{B} 2 / \mathrm{C} 2}$, corresponding to regions of low levels of polymorphism, could be exchanged with the corresponding sequence from BGT-51460 without loss of AVR function (swap $\# 6$ and \#8, Fig. 5b-d). Moreover, both chimeric proteins resulted in significantly stronger recognition by $\mathrm{PM} 3 \mathrm{~B}$ and $\mathrm{PM} 3 \mathrm{C}$ as compared to the natural sequence (Fig. $5 c, d$ ). In one additional swap (construct \#9), we simultaneously introduced segment a corresponding to the region with higher level of polymorphism, and segment $c$ from AVRPM3 $3^{\mathrm{B} 2 / \mathrm{C} 2}$ to BGT-51460. Consistent with the observed natural diversity, chimeric protein \#9 resulted in gain of AVR function by BGT-51460, and induced a much stronger recognition reaction by $\mathrm{PM} 3 \mathrm{~B}$ and $\mathrm{PM} 3 \mathrm{C}$ as compared to the natural sequence (Fig. 5e). This further supports the hypothesis that overall protein structure, conserved between members of effector families, as well as specific contact regions are important for PM3-dependent recognition of AVR proteins, and that natural diversity studies can be used to predict such regions.

The Pm3 alleles can recognise AVRs from non-adapted mildews. There has been accumulating genetic evidence suggesting that $A v r-R$ interactions are playing an important role as determinants of host-specificity in cereal mildews (Supplementary Note 7). Therefore, we aimed at investigating the role of the Pm3 alleles as possible determinants of host specificity to B.g. secalis and B.g. dactylidis, two mildew formae speciales only growing on rye (Secale cereale) or the wild grass Dactylis glomerata, respectively. We took advantage of the availability of the genome sequences of 5 isolates of rye mildew and 2 of Dactylis mildew to recover the sequences of the homologues of $A v r P m 3^{a 2 / f 2}$, $A v r P m 3 b 2^{b 2 / c 2}$ and $A v r P m 3^{d 3}$. We found homologues of all three AvrPm3 genes in B.g. dactylidis (hereafter referred to as $A v r P m 3^{a 2 /}$ ${ }^{2}-B g d, A v r P m 3^{b 2 / c 2}-B g d$, and $\left.A v r P m 3^{d 3}-B g d\right)$ and homologues of AvrPm $3 b^{b 2 / c 2}$ and $A v r P m 3^{d 3}$ in B.g. secalis $\left(A v r P m 3 b 2^{b 2 / c 2}-B g s\right.$, and $A v r P m 3^{d 3}-B g s$ ) (Supplementary Fig. 22a-c). We found no homologue of $A v r P m 3^{a 2 / f 2}$ in rye mildew. RNA-Seq data from two rye mildew isolates S-1391 and S-145938 shows that $A v r P m 3 b 2^{b 2 /}$ ${ }^{c}{ }^{-} B g s$ and $A v r P m 3^{d 3}-B g s$ are expressed during compatible interaction between B.g. secalis and rye (Supplementary Fig. 22d, e). All $A v r P m 3$ homologues were tested for $R$ gene recognition using the transient assay in $N$. benthamiana (Fig. 6a-c, Supplementary Fig. 23a-g). We observed no HR when AvrPm3 $3^{a 2 / f 2}-B g d$ was coexpressed with $P m 3 a$ or $P m 3 f$ (Fig. 6a). For $A v r P m 3^{b 2 / c 2}$, coexpression of the homologue from B.g. dactylidis with $P m 3 b$ and $P m 3 c$ resulted in no HR, while interestingly the homologue from B.g. secalis was recognised by both NLRs (Fig. 6b). Similarly, both $A v r P m 3^{d 3}$ homologues from rye and Dactylis powdery mildews were recognised by $P m 3 d$ (Fig. 6c). For AVRPM3 $32 / \mathrm{C} 2-\mathrm{BGD}$, protein expression data indicate that loss of recognition is not due to absence of the proteins (Supplementary Fig. 23j, k). However, data for AVRPM3 ${ }^{\mathrm{A} 2 / \mathrm{F} 2}$-BGD indicate this protein is low expressed in our assay (Supplementary Fig. 23h,i), therefore we cannot exclude that $A v r P m 3^{a 2 / f 2}-B g d-B g d$ is also recognised by $P m 3 a / f$ if expressed at higher levels.

The recognised AVRPM3 $3^{\mathrm{B} 2 / \mathrm{C} 2}-\mathrm{BGS}, \mathrm{AVRPM}^{\mathrm{D} 3}-\mathrm{BGS}$ and AVRPM $3^{\mathrm{D} 3}$-BGD homologues differ by 4,7 and 6 residues in the mature protein from their B.g. tritici encoded homologues respectively, demonstrating a higher level of sequence divergence as compared to the haplotypes of the AvrPm3 genes in B.g. tritici. Finally, we also tested possible recognition of the rye and Dactylis mildew AvrPm3 homologues by the rye powdery mildew resistance gene $P m 8$ in transient assays in $N$. benthamiana and observed no HR (Supplementary Fig. 24). Altogether, this data indicates strong conservation of AvrPm3 function and recognition specificity in grass mildews, and suggests that highly specific AvrPm3-Pm3 interactions are involved in defence against nonhost mildew forms.

To further substantiate these results, we designed a microphenomics assay to test for wheat susceptibility to non-host mildews in presence vs. absence of the Pm3 genes. Transgenic wheat lines expressing the $P m 3 b$ and $P m 3 d$ resistance alleles were infected with two isolates from the non-adapted rye powdery mildew (Bgs_1391 and Bgs_1459). As no macroscopic growth or sporulation of rye mildew can be observed in this non-host interaction, we microscopically phenotyped these assays for micro-colony formation, i.e., the ability of mildew to establish a haustorium and few secondary hyphae (see Methods). Transgenic lines were compared to the non-transgenic 'Bobwhite' genotype, i.e., the identical genetic background of the $P m 3 b$ and $P m 3 d$ transgenics. We found a significant reduction of the ability of the non-adapted rye powdery mildew to form micro-colonies on the transgenic lines as compared to the non-transgenic control (Fig. 6d). To further extend these observations, we performed the same assay using the $P m 3 b$ and $P m 3 c$ near isogenic wheat lines (NILs) 'Chul_8xChancellor' and 'Sonora_8xChancellor', respectively. The backcrossing line 'Chancellor' corresponding to the genetic background of the $P m 3 b$ and Pm3c NILs, was used as a control. In agreement with our first assay, we also found a significant reduction of micro-colony formation on the Pm3 NILs as compared to the control (Fig. 6e), further substantiating that the $P m 3 b, c, d$ alleles are acting as determinants of host specificity for non-adapted mildews. Finally, we also cloned and assayed the suppressor activity of the $\operatorname{SvrPm} 3^{a 1 / f 1}$ homologue encoded in the Bgs_1391 and Bgs_1459 genomes. We found these isolates encode for the previously described $S v r P m 3^{a 1 / f 1}-J$ haplotype, which differs by only one residue from the B.g. dacylidis sequence (Supplementary Fig. 25a). RNA-Seq data indicated the rye mildew encoded $\operatorname{SvrPm} 3^{a 1 / f 1_{-} J}$ is expressed at very low levels upon infection of rye (Supplementary Fig. 25b). Using transient assays in $N$. benthamiana, we could show that this variant is indeed an inactive suppressor comparable to the previously described SvrPm3 ${ }^{a 1 / f 1}$-C from the Pm3 avirulent wheat mildew isolate Bgt_96224 (Supplementary Fig. 25c).

We conclude that, (i) specific recognition of divergent AvrPm3 homologues from rye and Dactylis mildews by the Pm3b,c,d alleles, (ii) increased non-host resistance to rye-mildew isolates encoding active AvrPm3 homologues in presence of the Pm3 alleles, and (iii) presence of an inactive $S v r P m 3^{a 1 / f 1}$ suppressor variant in those same isolates, is strong evidence supporting the role of the $P m 3$ alleles as determinants of host-specificity in cereal mildews based on specific and conserved AvrPm3-Pm3 interactions.

\section{Discussion}

Allelic series of resistance genes are highly informative to molecularly characterise resistance function if they are studied both on the host and the pathogen side. Genetic work in the 1990s and more recent molecular analysis has suggested that allelic series of resistance genes with distinct specificities are very promising systems to dissect the molecular basis of complex, race-specific interactions of biotrophic fungal pathogens such as rusts or 
a

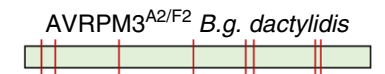

$\mathrm{AvrPm}^{3 \mathrm{a} 2 / 22}{ }_{-} \mathrm{Bgd} \mathrm{AvrPm}^{3 \mathrm{aa} / / 2}{ }_{-} \mathrm{Bgd}$ $+\mathrm{Pm} 3 \mathrm{a}$ $+\mathrm{Pm} 3 \mathrm{f}$
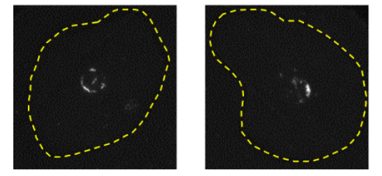

b

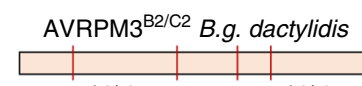
$\mathrm{AvrPm}^{\mathrm{b} 2 / \mathrm{c} 2}{ }_{\mathrm{Bgd}} \mathrm{AvrPm}^{\mathrm{b} 2 / \mathrm{c} 2}{ }_{\mathrm{Bgd}}$ $+\mathrm{Pm} 3 \mathrm{~b}$

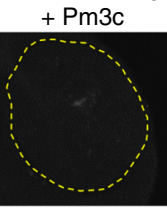

$\mathrm{AVRPM}^{\mathrm{B} 2 / \mathrm{C} 2}$ B.g. secalis

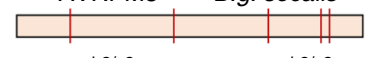

$\mathrm{AvrPm}^{\mathrm{b} 2 / \mathrm{c} 2}{ }_{-} \mathrm{Bgs} \quad \mathrm{AvrPm}^{\mathrm{b} 2 / \mathrm{c} 2}{ }_{-} \mathrm{Bgs}$ $+\mathrm{Pm} 3 \mathrm{~b}$
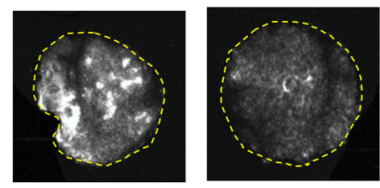

PMCS

РМЗА

PM3F

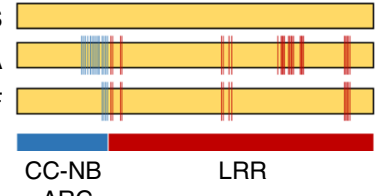

-ARC

d

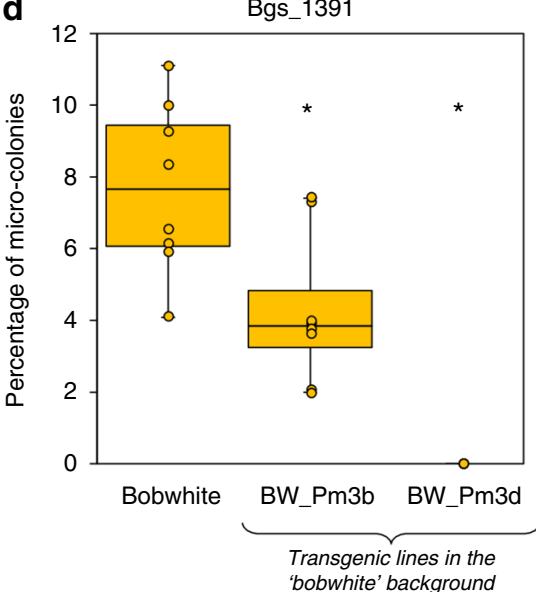

e

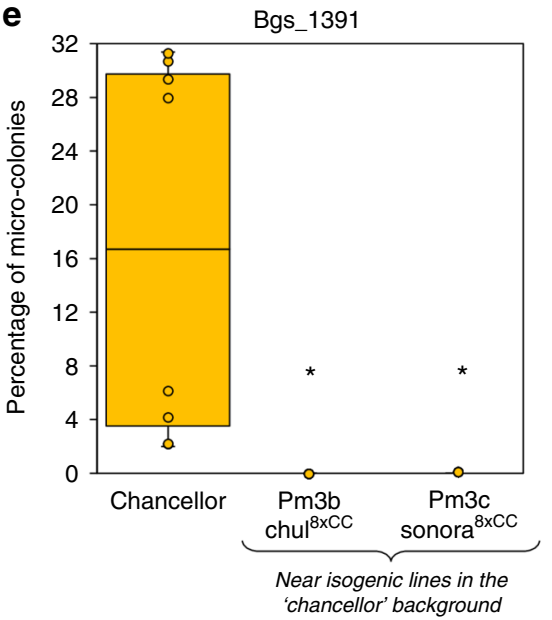

$\mathrm{PM} 3 \mathrm{C}$

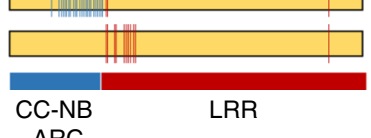

C

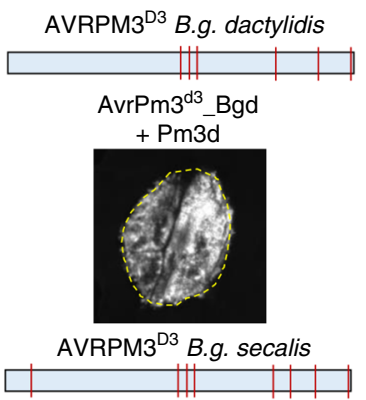

AvrPm3 ${ }^{\mathrm{d} 3}{ }_{-} \mathrm{Bgs}$ + Pm3d

PMCS

PM3D
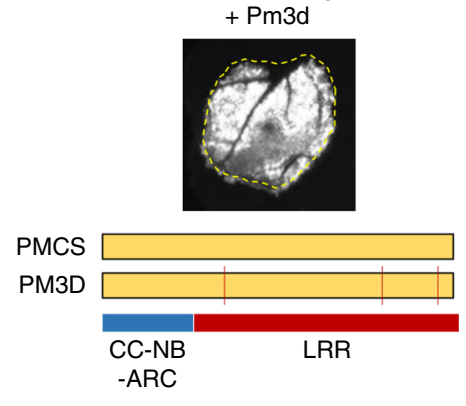

Bgs_1459
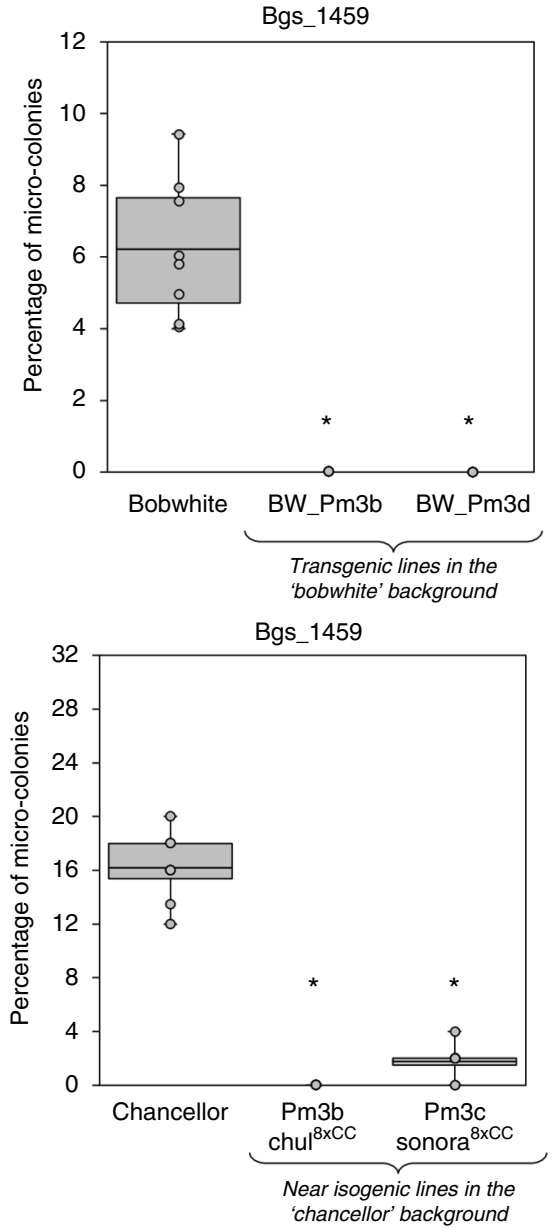

mildews with their hosts ${ }^{10}$. The analysis of recognition specificity in the cereal powdery mildew and the flax rust systems based on allelic series of resistance genes has revealed additional layers of complexity to the original gene-for-gene interaction model postulated by Flor ${ }^{11}$, because of the presence of suppressor/inhibitor genes and other modifiers of the interaction ${ }^{10}$. In addition to the Pm3 allelic series of 17 race-specific resistance alleles to wheat mildew $^{25}$, there are three other well studied examples of true allelic series of functionally different resistance specificities: the downy mildew (Hyaloperonospora arabidopsidis) gene RPP13 in 
Fig. 6 Conservation of AvrPm3-Pm3 recognition in grass mildews a-c Transient expression assays combining the identified grass mildew homologues of $\mathrm{AVRPM}^{\mathrm{A} 2 / \mathrm{F} 2}(\mathbf{a}), \mathrm{AVRPM}^{\mathrm{B} 2 / \mathrm{C} 2}(\mathbf{b})$, and $\mathrm{AVRPM}^{\mathrm{D} 3}(\mathbf{c})$, with their corresponding PM3 NLR receptors. Results are consistent over at least two independent assays each consisting of 6-8 independent leaf replicates. Complete N. benthamiana leaf pictures are provided in a Source Data File. Polymorphic residues compared to the B.g. tritici encoded sequences are schematically indicated with vertical bars. Similarly, polymorphic residues in the PM3 protein variants compared to the susceptible PM3CS sequence are indicated with vertical blue (CC-NB-ARC) and red (LRR) lines in the lower panel. d, e Percentage of micro-colonies formed by the non-adapted B.g. secalis isolates Bgs_1391 and Bgs_1459 on d transgenic lines expressing the Pm3b (BW_PM3b) and Pm3d (BW_Pm3d) resistance genes in the hexaploid wheat genotype 'Bobwhite', compared to the non-transgenic 'Bobwhite' control, e Near isogenic lines (NILs) expressing the $\mathrm{Pm} 3 b$ (Chul ${ }^{8 \times C C}$ ) and $\mathrm{Pm} 3 c$ (Sonora ${ }^{8 \times C C}$ ) resistance loci backcrossed 8 times with the hexaploid wheat genotype 'Chancellor', compared to a 'Chancellor' control. Both 'Bobwhite and 'Chancellor' are fully susceptible to adapted B.g. tritici races. Micro-colony formation was scored microscopically 2 days post rye mildew infection (see Methods). Values are representative of the average from eight independent leaf replicates. Mean values are indicated by the middle line in the boxplot. Individual data points are plotted along the whiskers delineating minimum and maximum values. Differences to the control were statistically tested with the Wilcoxon Rank Sum Test. Statistical significance $(p<0.05)$ is indicated with a star. Raw data underlying the reported averages are provided in a Source Data File

Arabidopsis thaliana ${ }^{39}$, the flax rust (Melampsora lini) resistance gene $L^{22}$, and the Mla genes in barley (Hordeum vulgare) ${ }^{27}$. The Pm3 alleles stand-out with a particularly high level of sequence conservation ( $>97 \%$ identity at the protein level) ${ }^{25,40}$, while the Mla alleles have $84.6 \%$ sequence identity and are as divergent as the Arabidopsis RPP13 and the flax $L$ allelic variants ${ }^{22,27,39}$.

Whereas several allelic variants of RPP13 and the flax rust NLRs L5, L6 and L7 detect allelic AVR proteins, there is evidence that additional members of these allelic series also recognise sequence unrelated effectors $23,41,42$. This principle is further exemplified by MLA1 and MLA13, which show $>91 \%$ identity on the protein level but recognise the sequence unrelated $B g h$ proteins $\mathrm{AVR}_{\mathrm{A} 1}$ and $\mathrm{AVR}_{\mathrm{A} 13}{ }^{31}$. Consistent with these findings, the so far identified AVR proteins of the Pm3 allelic series AVRPM $^{\mathrm{A} 2 / \mathrm{F} 2}$, AVRPM3 ${ }^{\mathrm{B} 2 / \mathrm{C} 2}$ and AVRPM3 $3^{\mathrm{D} 3}$ are members of three distinct B.g. tritici effector gene families with low sequence identity, and belong to large groups of phylogenetically related effectors with a possible common origin (in particular $\mathrm{AVRPM}^{\mathrm{A} 2 / \mathrm{F} 2}$ and AVRPM3 ${ }^{\mathrm{B} 2 / \mathrm{C} 2}$ ) (Supplementary Figs. 11 and 13). Together, phylogenetic relatedness, positional conservation of amino-acid with similar properties and structural residues across AVRPM3 families (Supplementary Fig. 12), and possible similarities of tertiary protein folds (Supplementary Fig. 14), suggests that specificity of the Pm3 alleles is possibly based on recognition of structurally similar effectors. This is reminiscent of the identification of diverse, but structurally conserved MAX effectors in different phytopathogenic fungi ${ }^{43}$, and would suggest it is plausible that AVRPM3 ${ }^{\mathrm{D} 3}$, which stands out phylogenetically, would also share the same structure as AVRPM3 ${ }^{\mathrm{A} 2 / \mathrm{F} 2}$ and $A V R P M 3^{B 2 / C 2}$. Here, the detection of structurally similar proteins might provide the plant with a crucial benefit in an evolutionary arms race with rapidly evolving fungal proteins, which are restricted only by the requirement to conserve important virulence functions that might be mainly dependent on protein structure. Thus, the AVRPM3-PM3 interactome might provide a highly suitable model for the study of rapid protein sequence evolution in eukaryotes under possibly the only constraint of structural conservation.

Recent studies of effector functions in barley powdery mildew have established the role of effectors as important virulence factors (reviewed in ref. ${ }^{14}$ ) that have the ability to interfere with components of the host basal metabolism and host immunity ${ }^{16-19}$. High frequency of active AvrPm3 haplotypes among wheat mildew populations, and the fact that homologues can also be found in the related formae speciales B.g. secalis and B.g. dactylidis, suggest that all $A v r P m 3$ genes are important for mildew virulence. Despite strong indication of diversifying selection on $A v r P m 3^{b 2 / c 2}$ and as previously shown on $A v r P m 3^{a 2 / f 229}$, no presence-absence polymorphisms or transposable element insertions could be detected for $A v r P m 3^{a 2 / f 229}$, or $A v r P m 3^{b 2 / c 2}$ (this study) in 272 and
185 B.g. tritici isolates respectively. Even in the case of $A v r P m 3^{d 3}$ with the avirulent version being absent in numerous isolates, no cases of complete loss of the effector could be identified, thus indicating that the AvrPm3 effectors are always present together in all wheat powdery mildew isolates globally. This stand in strong contrast to AvrPm2, $A v r_{a 1}, A v r_{a 13}$ and AvrPib from B.g. tritici, B.g. hordei and Magnaporthe oryzae, respectively, where loss of avirulence was exclusively $(A v r P m 2)$, predominantly $(A v r P i b)$ or partially ( $A v r_{a 1}$ and $\left.A v r_{a 13}\right)$ found to be dependent on presence/ absence polymorphisms, gene disruptions by transposable element insertions, and segmental deletions ${ }^{30,31,44}$. These findings suggest there is a selective advantage for the pathogen to simultaneously encode all three effectors. We suggest that the inability to completely lose these effector genes is compensated partially by the identified diversifying selection, giving rise to new, unrecognised variants, and in parts by the presence and activity of $\operatorname{SvrPm} 3^{a 1 / f 1}$ that allows the wheat powdery mildew fungus to mask, and preserve effectors that would otherwise be recognised by the Pm3 alleles. Previous studies in a few mildew isolates indicated that suppression of $A v r P m 3$ recognition is only effective when the active $S v r P m 3^{a 1 / f 1}$ gene is highly expressed ${ }^{28,29}$. In this study, we did not determine the presence and expression levels of SvrPm $3^{a 1 / f 1}$ in the GWAS population. We propose that future integration of transcriptome data in genetic association and population genomics studies will provide important information on the role of $S v r P m 3^{1 / f 1}$ in controlling both race-specificity and host-specificity in global cereal mildew populations. For AVRPM $^{\mathrm{B} 2 / \mathrm{C} 2}$, domain swap studies identified two regions with higher levels of polymorphism in the mildew population that are sufficient for conferring AVR function, and two additional segments quantitatively affecting the strength of AVR recognition. McNally and colleagues ${ }^{29}$, also found that specific domains of AVRPM3 $3^{\mathrm{A} 2 / \mathrm{F} 2}$ are involved in immune receptor recognition, including one region quantitatively affecting the strength of AVR recognition. We suggest these domains are possibly acting as contact regions affecting AVR-R recognition and binding affinity, which is reminiscent of the identification of 4 residues, spanning a 21 amino-acid region in the rice blast avirulence protein AVRPik and affecting recognition and binding affinity to rice Pik resistance protein ${ }^{45}$

The evolutionary history of grass powdery mildews has been reconstructed recently. Menardo and colleagues ${ }^{13}$ found that the radiation of the B.g tritici-clade, consisting of B.g. tritici, B.g. secalis and B.g. dactylidis has occurred 170,000 to 280,000 years ago and involved a host jump from wheat to Dactylis glomerata, a wild grass species, giving rise to B.g. dactylidis. The recent radiation of the tritici-clade is also reflected in their respective Avr-effector complement. Of the four Avr genes that have been identified in B.g. tritici, homologues can also be found in either B.g. secalis (AvrPm2), B.g. dactylidis (AvrPm3 $3^{a 2 / f 2}$ ) or both closely 
related formae speciales $\left(A v r P m 3^{b 2 / c 2}, A v r P m 3^{d 3}\right)$. Furthermore, all $A v r$ homologues from B.g. secalis and the AvrPm $3^{d 3}$ homologue from B.g. dactylidis are recognised by the corresponding Pm3 alleles from wheat ${ }^{30}$ (this study). The recognised variants of AvrPm $3^{b 2 / c 2}$ from B.g. secalis and $A v r P m 3^{d 3}$ from B.g. secalis and B.g. dactylidis contain 4, 7 and 6 non-synonymous SNPs in the mature peptide, respectively, when compared to the $P m 3$-recognised B.g. tritici variants. This stands in strong contrast to the rather limited $A v r P m 3$ variation found within wheat mildew populations, where single non-synonymous mutations often result in evasion of recognition. We hypothesise that this reflects the fact that the currently active $P m 3$ alleles have evolved only after wheat domestication $\sim 11,000$ years ago ${ }^{24}$, and the AvrPm3 homologues in closely related formae speciales have therefore not been targets of diversifying selection due to presence of the Pm3 alleles in corresponding rye or Dactylis host species.

The presence of the Pm3 alleles does severely restrict, often completely abolish the formation of non-sporulating microcolonies by the non-adapted rye mildew (Fig. 6d, e). This is consistent with a previous study using a genetic cross between wheat and rye powdery mildews that has predicted over 20 years ago the presence of $A v r P m 2, A v r P m 3^{b}$ and $A v r P m 3^{c}$ in the B.g. secalis genome, and suggested their role as host-specify determinant ${ }^{46}$. The ability of non-host mildews to establish microcolonies on susceptible wheat cultivars might provide a basis for mating with adapted mildew isolates, thus giving raise to new hybrid forms with a broader host range such as Triticale powdery mildew ${ }^{47}$. Recent work by Praz and colleagues provided transcriptional evidence that the ability of Triticale mildew to grow on wheat and its new host triticale is based on downregulation (i.e. transcriptional removal) of numerous effectors mainly encoded within AVR gene families ${ }^{38}$. Therefore, loss of $A v r$ function in grass mildew, combined with the deployment of susceptible wheat genotype (i.e. lacking $P m$ resistance genes), might provide a genetic basis for the raise of new adapted mildew forms. This hypothesis is substantiated by recent work by Inoue and colleagues $^{48}$, showing that the emergence of the wheat blast pathogen is due to widespread deployment of rwt3 wheat (i.e., lacking the $R w t 3$ resistance genes), combined with the loss of function of PWT3 (the Avr of Rwt3) in non-adapted forms of Pyricularia oryzae (syn. Magnaporthe oryzae), resulting in a host jump on wheat ${ }^{48}$. These findings suggest that major $R$ genes such as $P m 2$ and $P m 3$ must be maintained in the breeding gene pool even if they are locally defeated by virulent races from the adapted wheat mildew, because they are conferring important non-host resistance preventing the emergence of new mildew pathogens.

Based on these findings, we propose that the ability of other formae speciales of the tritici-clade to grow on wheat is restricted by the presence of many $A v r-R$ gene pairs. These are simultaneously involved in race-specific resistance and in host specificity, similar to more generalised models proposed by (i) Tosa ${ }^{49}$, who argued that that the acquisition or loss of Avr genes is the main driving force behind the evolution of new formae speciales, a hypothesis recently substantiated by findings from wheat and rice blast ${ }^{48,50}$, and (ii) Schulze-Lefert and Panstruga (2011) ${ }^{51,52}$ suggesting that the contribution of major $R$-genes to non-host resistance inversely correlates with the phylogenetic divergence time between the analysed host and non-host plants of a certain pathogen species or formae speciales.

\section{Methods}

Fungal isolates and virulence tests. Blumeria graminis $\mathrm{f}$. $\mathrm{sp}$. tritici isolates were maintained in the asexual phase on detached leaves of the susceptible bread wheat (Triticum aestivum) cultivar Kanzler on 0.5\% food grade agar (PanReac AppliChem) plates supplemented with $4.23 \mathrm{mM}$ benzimidazole ${ }^{53}$. Virulence tests were performed on near-isogenic lines or varieties using 'Asosan $/ 8^{\star}$ Chancellor' for $P m 3 a$, 'Chul/8xChancellor' for $P m 3 b$, 'Sonora/8xChancellor' for $P m 3 c$, 'Kolibri' for Pm3d, 'W150' for Pm3e, 'Michigan Amber/8xChancellor' for Pm3f and the susceptible wheat cultivars 'Kanzler' and 'Chancellor' as a control ${ }^{35}$. Virulence scoring was performed 10 days after infection on at least three independent leaf segments. Scoring was performed qualitatively as follows: mildew leaf coverage (LC) of $60-100 \%$, virulent; LC of $10-40 \%$, intermediate; LC of $0 \%$, avirulent ${ }^{28}$.

For the GWAS, a population of natural isolates was established by collecting mildew races from infected wheat leaves in the fields of major mildew epidemic regions in China from 2011 to 2014 described by Zeng and collaborators ${ }^{54}$. Singlepustule-derived isolates were purified by growing the pathogen on the susceptible wheat line 'Chancellor,' then multiplied and stored at $-80^{\circ} \mathrm{C}$ by drying spores at $23{ }^{\circ} \mathrm{C}$ for $5 \mathrm{~h}$ in the presence of silica gel before freezing 55 . One hundred pure isolates with diversified geographic origin and a balanced virulence/avirulence pattern on wheat differential resistance lines were manually selected from the natural population (Supplementary Data 2).

Blumeria graminis $\mathrm{f}$. sp. secalis isolates S-1391 and S-1459 were maintained in the asexual phase on detached leaves of the susceptible rye (Secale cereale) cultivar 'Matador' on $0.5 \%$ food grade agar (PanReac AppliChem) plates supplemented with $4.23 \mathrm{mM}$ benzimidazole ${ }^{38}$. To assess the influence of the $P m 3 b, P m 3 c$ and $P m 3 d$ alleles on early stages of infection on wheat, detached primary leaves of near-isogenic wheat lines 'Chul/8xChancellor' for $\mathrm{Pm} 3 b$, 'Sonora/8xChancellor' for $P m 3 c$, transgenic wheat lines $\mathrm{Pm} 3 \mathrm{~b} \# 1^{56}, \mathrm{Pm} 3 \mathrm{~d} \# 1^{57}$ and the corresponding susceptible control 'Chancellor' and 'Bobwhite' were infected with S-1391 and S-1459 as described above for B.g. tritici. Leaf segments were stained $48 \mathrm{hpi}$ for reactive oxygen species in $1 \mathrm{mg} \mathrm{ml}^{-1} 3^{\prime} 3$-diaminobenzidine (DAB)- $\mathrm{HCl}$, $\mathrm{pH} 3.8$ solution for $12 \mathrm{~h}^{58}$ followed by complete de-staining of leaf pigments in ethanol:acetic acid solution (ratio 3:1) for several days. To detect fungal spores and hyphae the destained leaf segments were subsequently stained with Coomassie Brilliant Blue $\left(0.15 \%\right.$ in EtOH absolute), washed repeatedly in $\mathrm{H}_{2} \mathrm{O}$ and samples mounted for microscopy in $50 \%$ glycerol. Using a conventional bright-field microscope, spores were scored based on the following categories: (i) microcolony formation: establishment of a haustorium and production of secondary hyphae without apparent signs of hypersensitive cell-death (HR) indicating a compatible interaction in early stages of infection, (ii) penetration of epidermal cells resulting in HR and stop of growth, or early arrest of spore growth in the absence of HR. For each isolate/cultivar combination the average of eight biological replicates (=independent leaf segments) is shown. For each leaf segment at least 50 spores were assessed. The assay was repeated with similar results. Statistical significance of observed differences was tested using a Wilcoxon rank sum test for possibly tied observation (wilcox.exact function, R package exactRankTests).

Haplotype mining. Haplotype diversity was assessed on a subset of the worldwide B. graminis $\mathrm{f}$. sp. tritici collection previously described by $\mathrm{McNally}$ and colleagues $^{29}$. For the isolates originating from the United States, Israel, and Europe the AvrPm3 $3^{b 2 / c 2}$ and $A v r P m 3^{d 3}$ genes were PCR amplified from genomic DNA with primers listed in Supplementary Table 1, and Sanger-sequenced. All other isolates, including B.g. secalis and B.g. dactylidis accessions, were mined for the same two genes using genome sequence data ${ }^{13,30,47}$. Coding sequences of $A v r P m 3^{b 2 / c 2}$ variants in B.g. tritici, B.g. secalis and B.g. dactylidis found in this study are available under GenBank accession numbers MK806469-MK806484. Estimates for pN/pS ratio $^{59}$ of $A v r P m 3^{b 2 / c 2}$ haplotypes were calculated using the DnaSP 6 software package with default parameters ${ }^{60}$. Estimation of copy number variation of $A v r P m 3^{b 2 / c 2}$, and $A v r P m 3^{d 3}$ and its paralog BgtE-20069a was visualised based on a previously published analysis of gene coverage in 36 wheat powdery mildew genomes by Müller and colleagues ${ }^{34}$.

DNA/RNA isolation. Fungal high molecular weight DNA was extracted using an optimised procedure described in detail by Bourras and colleagues ${ }^{28,30}$. In short, $\sim 100 \mathrm{mg}$ of conidia were frozen in liquid nitrogen and ground three times $30 \mathrm{~s}$ using stainless steel beads and a high-speed plate grinder (MM200 Mixer Mill, Retsch, Germany) at a grinding frequency of $30 \mathrm{~s}^{-1}$. To the ground conidia, $300 \mu \mathrm{l}$ of $65^{\circ} \mathrm{C}$ preheated $5 \%$ Sarcosyl solution was added and vigorously vortexed. After addition of $700 \mu \mathrm{l}$ of CTAB-Buffer $(0.2 \mathrm{M}$ Tris(hydroxymethyl)aminomethane at pH 7.5, 50 mM EDTA, $2 \mathrm{M} \mathrm{NaCl}, 2 \%$ Cetyl trimethylammonium bromide (CTAB) and $0.25 \mathrm{M}$ Sodium metabisulfite (Na2S2O5)) the samples were incubated at $65^{\circ} \mathrm{C}$ for $30 \mathrm{~min}$. After incubation, $600 \mu \mathrm{l}$ Chloroform was added, followed by $10 \mathrm{~min}$ of centrifugation at $14,000 \mathrm{rcf}, 4^{\circ} \mathrm{C}$. The supernatant was collected and combined with one volume of $-20^{\circ} \mathrm{C}$ precooled Isopropanol, mixed by careful inversion and centrifuged for $10 \mathrm{~min}, 14,000 \mathrm{rcf}, 4^{\circ} \mathrm{C}$. The resulting pellet was dried on ice and resuspended in standard TrisEDTA buffer before further purification on AmiconUltra $0.5 \mathrm{ml}$ centrifugal filters MWCO $30 \mathrm{kDa}$ (Sigma-Aldrich, Steinheim, Germany).

RNA samples were extracted from infected wheat leaves of the susceptible cultivar 'Chinese Spring' using the SV Total RNA Isolation System (Promega) according to the manufacturer's protocol. RNA integrity was assessed by gel electrophoresis and spectrophotometric analysis using a NanoDrop 1000 Sepctrophotometer (ThermoScientific) ${ }^{38}$. RACE-PCR was performed using the SMARTer RACE cDNA Amplification Kit (Clontech Laboratories Inc.) according to the manufacturer and with gene-specific primers listed in Supplementary Data 4. 
Codon optimisation, gene synthesis and plasmids. Codon optimisation of effector coding sequences for expression in $N$. benthamiana was performed using the online tool provided by Integrated DNA technologies (https://eu.idtdna.com/ CodonOpt). In all cases the signal peptide, as predicted by the SignalP4 algorithm ${ }^{61}$ (http://www.cbs.dtu.dk/services/SignalP/) was replaced by an ATG start codon. Gene synthesis including gateway-compatible attL cloning sites was performed with commercial partners: Gen9 (https://www.gen9bio.com), BioCat (https://www. biocat.com), Invitrogen (https://www.thermofisher.com) and Twist Bioscience (https://twistbioscience.com). The synthesised genes were cloned into the $A$. tumefaciens expression vector pIPKb004 ${ }^{62}$ using Gateway LR clonase II (Invitrogen) according to the manufacturer and subsequently transformed into A. tumefaciens strain GV3101 using electroporation $(1.44 \mathrm{kV}, 25 \mu \mathrm{F}, 200 \Omega)^{28}$.

Site-directed mutagenesis (SDM) was performed by PCR amplification on primary gene-synthesis products with non-overlapping primers (Supplementary Table 2). Linearised SDM products were phosphorylated using T4 polynucleotide kinase (New England Biolabs) and re-ligated with T4 DNA Ligase (New England Biolabs) according to the manufacturer. Epitope tagged Pm3 alleles have been published by Brunner and colleagues ${ }^{56}$. Epitope tagging of effector genes has been performed by gene-synthesis or SDM as described above (see also Supplementary Note 3). A complete list of all synthesised or mutagenized (SDM) constructs including nucleotide sequence and manufacturer can be found in Supplementary Data 3.

BAC sequencing. The B.g. tritici BAC library has been previously produced from the reference isolate Bgt_96224 and is described in ${ }^{53}$. BACs were selected based on their positions in BAC fingerprint assemblies generated with LTC $^{63}$ and manually combined into a scaffold (Supplementary Fig. 2, Supplementary Table 3). Candidate clones were confirmed by PCR and plasmids were extracted using the Qiagen Large-Construct Kit according to the manufacturer's protocol. BAC insert sizes were estimated by digestion with NotI and pulsed field gel electrophoresis. Selected BAC clones were sequenced using Illumina MiSeq technology $(2 \times 250$ bp paired end; GATC Biotech). Individual BAC reads were assembled using the CLC Genomics Workbench version 6.0.1 with default settings.

RNA-seq data analysis. Gene expression data at 2 dpi on the susceptible cultivar 'Chinese Spring' for the B.g. tritici isolates Bgt_96224,_94202, and_JIW2 was obtained from the study of Praz and colleagues ${ }^{38}$ available at Gene Expression Omnibus (GEO) under accession number GSE108405 [https://www.ncbi.nlm.nih. gov/gds/?term=GSE108405]. Reads per kilo base pairs per million reads (rpkm) values were obtained with edge $\mathrm{R}^{64}$ and used for the effector benchmarking analysis. Normalisation was done with edgeR using the command calcNormFactors(method = "TMM"). Genes were considered as expressed if the cpm (count per million) was $>10$ in at least three of the nine replicates. Genes were considered as differentially expressed if the $\log 2$ fold change $(\log 2 \mathrm{FC})$ value obtained with edgeR was $>1$. Statistical analysis was performed with edgeR using the following sequence of commands: estimateGLMCommonDisp(), estimateGLMTrendedDisp(), estimateGLMTagwiseDisp(), glmFit() and glmLRT(). RNA-Seq data of three B.g. tritici and two B.g. secalis isolates (obtained from GSE108405, three replicates each) were also mapped on the new PacBio reference genome assembly (v3.16 $\left.{ }^{34}\right)$ using STAR ${ }^{65}$ with the following parameters:-outFilterMultimapNmax

10-outFiltermismatchNoverLmax 0.02-alignIntronMax 500. We obtained expression counts for each gene with the most recent gene annotation from Müller and colleagues $^{34}$ using Salmon with standard parameters ${ }^{66}$.

Genome-wide association study. Fungal isolates used for GWAS are described in Supplementary Data 2. Genomic sequences are available under the accession number SRP062198 [https://www.ncbi.nlm.nih.gov/sra/?term = SRP062198] (from ${ }^{30}$ and this study). Illumina reads were mapped on the reference genome (v3.16) using Bowtie2 with parameters $-\mathrm{L},-0.6,-0.25^{67}$. The resulting Bam files were processed with the SAMtools view and sort commands ${ }^{68}$. Duplicated reads were removed with the SAMtools command rmdup. Picard Tools was used to add read groups to the bam files (http://broadinstitute.github.io/picard). Bam files were merged using bamtools merge command and indexed using samtools index command. SNP calling was done using freebayes ${ }^{69}$ with the following parameters: -p 1 -m 30 -q 20 -z 0.03 -F 0.7-3 200-genotype-qualities. VCF file was subsequently filtered using vcftools with the following parameters:-maf $0.05-\max -$ meanDP 40-remove-indels-min-alleles 2-max-alleles 2-minGQ 30-max-missing 0.9 . VCF file was transformed into hapmap format using a custom perl script. GWAS was performed using the GAPIT package for $\mathrm{R}^{33}$ using the the PCA.total = 3 option.

Transient assays in Nicotiana benthamiana and HR measurement. A. tumefaciens mediated transient expression of AvrPm 3 candidates, SvrPm 3 and $P m 3$ alleles in N. benthamiana was achieved as follows: Agrobacteria were grown in Luria broth (LB) medium supplemented with appropriate antibiotics overnight at $28^{\circ} \mathrm{C}$ with $200 \mathrm{rpm}$ shaking. Fully grown cultures were harvested by centrifugation $3300 \times g, 5 \mathrm{~min}$, resuspended in fresh LB medium without antibiotics and further incubated for $30 \mathrm{~min}$ at $28^{\circ} \mathrm{C}$ with $200 \mathrm{rpm}$ shaking. Bacteria were harvested again by centrifugation at $3300 \times \mathrm{g}$ for $5 \mathrm{~min}$ and the pellet was resuspended and diluted in
AS-medium (10 mM MES-KOH, pH5.6; $10 \mathrm{mM} \mathrm{MgCl}_{2} ; 200 \mu \mathrm{M}$ acetosyringone) to an OD of 1.2 before incubation for 3 to $4 \mathrm{~h}$ at $28^{\circ} \mathrm{C}$ with $200 \mathrm{rpm}$ shaking to induce virulence ${ }^{28}$. Before infiltration into N. benthamiana, Agrobacteria expressing AvrPm 3 candidates, $S v r P m 3$ or $P m 3$ alleles were mixed in the indicated ratios.

HR was assessed visually and by fluorescence scanning using the Fusion FX Imaging System (Vilber Lourmat, Eberhardzell, Germany) with the following pre-settings: fluorescence sample; excitation: blue epi-illumintaion; emission: filter F-535 Y2; aperture: 0.84-open; sensitivity: full resolution ${ }^{30}$. The time-point of HR assessment was chosen depending on the experimental setup: 5 days after Agrobacterium infiltration for verification of AVR recognition by the corresponding R-gene, 2dpi for suppression assays involving $\operatorname{SvrPm} 3$ and to test for inter-allelic suppression. HR was quantified using high-resolution epifluorescence images obtained with the FusionFX HRS technology, and analysed with the Image image analysis software measuring the integrated density of fluorescence $e^{29,30}$. HR values were normalised to the infiltrated leaf area and to the control. Statistical significance was assessed with the Student's $t$-test for paired data $(p<0.05)$.

Protein detection. The soluble protein fraction was extracted from A.tumefaciens infiltrated $N$. benthamiana leaves 2 days after infiltration. Two leaf discs $(0.5 \mathrm{~mm}$ diameter) from 4 infiltrated leaves were pooled, ground in liquid nitrogen and resuspended in 2x modified Laemmli-Buffer (100 mM Tris-HCl pH 6.8, $200 \mathrm{mM}$ DTT, $0.04 \%$ bromphenol blue, $20 \%$ glycerol, $2 \%$ SDS), boiled for $5 \mathrm{~min}$ and centrifuged at $10,000 \times g$ for $10 \mathrm{~min}$ at $4{ }^{\circ} \mathrm{C}$. Ten microlitre of total protein extract was separated in SDS polyacrylamide (PA) gels (8\% PA for PM3-proteins, 16\% PA for effectors) and semi-dry blotted to a nitrocellulose membrane (Amersham Protran $0.2 \mu \mathrm{m} \mathrm{NC)}$ using a Trans-Blot SD Semi-Dry Transfer Cell (Bio-Rad). Blotted protein was stained with Ponceau S. For HA detection, a peroxidase conjugated antibody (anti-HA-HRP, rat monoclonal, clone 3F10, Roche) was used at a dilution of 1:3000. For FLAG detection the primary antibody (anti-FLAG, mouse monoclonal, clone M2, Sigma-Aldrich) was used at a dilution of 1:10,000, membranes washed with TBST and incubated with anti-mouse peroxidase antibody (anti-mouse-HRP, goat polyclonal, Sigma Aldrich) diluted 1:4000. Peroxidase chemiluminescence was detected using a Fusion FX Imaging System (Vilber Lourmat, Eberhardzell, Germany) and WesternBright ECL HRP substrate (Advansta).

Phylogenetic analysis and structural modelling. Protein sequences of the AVRPM $^{\mathrm{A} 2 / \mathrm{F} 2}, \mathrm{AVRPM}^{\mathrm{B} 2 / \mathrm{C} 2}, \mathrm{AVRPM}^{\mathrm{D} 3}$, SVRPM3 $3^{\mathrm{A1} / \mathrm{F1}}$ and the E005 effector families were obtained from Müller and colleagues ${ }^{34}$. Protein sequence alignments were performed using the MUSCLE v3.8 available online (https://www.ebi.ac.uk/ Tools/msa/muscle/), assessment of phylogenetic relationships, and construction/ depiction of the phylogenetic tree was performed using CLC workbench software (v7.9.1). We used the following parameters for multiple sequence alignments in CLC: gap open $\cos t=20.0$, gap extension $\operatorname{cost}=2.0$, end gap $\operatorname{cost}=$ as any other, alignment $=$ very accurate. We used the following parameters for tree construction: tree construction method $=$ UPGMA, protein distance measure $=$ Jukes-Cantor, bootstrapping replicates $=100$. Phylogenetic trees were depicted as circular phylograms with a threshold for bootstrap value $>80$, using CLC workbench (v7.9.1). Prediction of secondary protein folds was done with Quick2D (https://toolkit.tuebingen.mpg.de/ \#/tools/quick2d) an integrative prediction tool from the MPI Bioinformatics Toolkit $^{36}$. Briefly, sequences of the AVRPM3 proteins were submitted without the signal peptide, and secondary structure modelling was done with the recommended default parameters (database for MSA generation $=\mathrm{nr} 90$; maximal number of MSA generation steps $=3$; threshold $\mathrm{E}$-value $=1 \mathrm{e}-3$ ). The Quick2D modelling employs four different algorithms for prediction, namely a PSI-blast based secondary structure prediction algorithm (PSIPRED), an iterative deep neural network (DNN) based algorithm (SPIDER2), a multiple backpropagation neural network predictor (PSSPRED), and a deep convolutional neural fields based method (DEEPCNF). Secondary folds depictions were extracted and adapted from the Quick2D output. Three dimensional protein modelling was performed using the RaptorX protein modelling tool (http://raptorx.uchicago.edu/). Protein sequences corresponding to the predicted mature protein after cleavage of the predicted signal peptide were modelled. The resulting predicted folds were manually inspected and visualised in order to identify possible common structures. For each predicted fold, the $P$-value, the Global Distance Test (GDT) score, and uSeqID identity score were recorded (Supplementary Data 5). Detailed definitions of these score values are available in the RaptorX user documentation (http://raptorx.uchicago.edu/documentation/).

Reporting summary. Further information on research design is available in the Nature Research Reporting Summary linked to this article.

\section{Data availability}

Gene expression data was obtained from Gene Expression Omnibus (GEO) under accession number GSE108405 [https://www.ncbi.nlm.nih.gov/gds/?term = GSE108405] Genomic sequences of the fungal isolates used for GWAS are available under the accession number SRP062198 [https://www.ncbi.nlm.nih.gov/sra/?term = SRP062198]. Coding sequences of naturally occurring variants of $A v r P m 3^{b 2 / c 2}$ were deposited under 
Genbank accession numbers MK806469-MK806484 [https://www.ncbi.nlm.nih.gov/ nuccore/?term $=$ MK806469]. Individual gene and protein sequences used in this study are provided in Supplementary Data 3. The source data underlying Figs. 1(d, h), 2(a, b), 3 $(\mathrm{a}-\mathrm{g}), 4(\mathrm{a}, \mathrm{c}), 5(\mathrm{c}-\mathrm{e})$, and $6(\mathrm{a}-\mathrm{e})$ are provided as Source Data file. The source date underlying Supplementary Figs. 6(a-d), 7(a-d), 8(a, b), 9(a-e), 15, 18, 19(b), 20(c), 21(b), 22(d, e), 23(h-k), and 25(b, c) are provided as Source Data file. Any additional data or biological material that support the findings of this study are available from the corresponding author upon reasonable request.

Received: 3 May 2018 Accepted: 3 May 2019

Published online: 23 May 2019

\section{References}

1. Farrer, R. A. et al. Multiple emergences of genetically diverse amphibianinfecting chytrids include a globalized hypervirulent recombinant lineage. Proc. Natl Acad. Sci. USA 108, 18732-18736 (2011).

2. Blehert, D. S. et al. Bat white-nose syndrome: an emerging fungal pathogen? Science 323, 227 (2009).

3. Kim, K. \& Harvell, C. D. The rise and fall of a six-year coral-fungal epizootic. Am. Nat. 164, S52-S63 (2004).

4. vanEngelsdorp, D. et al. Colony collapse disorder: a descriptive study. PLoS One 4, e6481 (2009).

5. Jessup, D. A. et al. Southern Sea Otter as a Sentinel of Marine Ecosystem Health. Ecohealth 1, 239-245 (2004).

6. Cruz, C. D. \& Valent, B. Wheat blast disease: danger on the move. Trop. Plant Pathol. 42, 210-222 (2017).

7. Singh, R. P. et al. The emergence of Ug99 races of the stem rust fungus is a threat to world wheat production. Annu. Rev. Phytopathol. 49, 465-481 (2011).

8. Saintenac, C. et al. Identification of wheat gene Sr35 that confers resistance to Ug99 stem rust race group. Science 341, 783-786 (2013).

9. Salcedo, A. et al. Variation in the AvrSr35 gene determines Sr35 resistance against wheat stem rust race Ug99. Science 358, 1604-1606 (2017).

10. Bourras, S., McNally, K. E., Müller, M. C., Wicker, T. \& Keller, B. Avirulence genes in cereal powdery mildews: the gene-for-gene hypothesis 2.0. Front. Plant Sci. 7, 241 (2016).

11. Flor, H. Current status of the gene-for-gene concept. Annu. Rev. Phytopathol. 9, 275-296 (1971).

12. Troch, V. et al. Formae speciales of cereal powdery mildew: Close or distant relatives? Mol. Plant Pathol. 15, 304-314 (2014).

13. Menardo, F., Wicker, T. \& Keller, B. Reconstructing the evolutionary history of powdery mildew lineages (Blumeria graminis) at Different Evolutionary Time Scales with NGS data. Genome Biol. Evol. 9, 446-456 (2017).

14. Bourras, S., Praz, C. R., Spanu, P. D. \& Keller, B. Cereal powdery mildew effectors: a complex toolbox for an obligate pathogen. Curr. Opin. Microbiol. 46, 26-33 (2018).

15. Lo Presti, L. et al. Fungal effectors and plant susceptibility. Annu. Rev. Plant Biol. 66, 513-545 (2015).

16. Ahmed, A. A., Pedersen, C. \& Thordal-Christensen, H. The barley powdery mildew effector candidates CSEP0081 and CSEP0254 promote fungal infection success. PLoS ONE 11, e0157586 (2016).

17. Ahmed, A. A. et al. The barley powdery mildew candidate secreted effector protein CSEP0105 inhibits the chaperone activity of a small heat shock protein. Plant Physiol. 168, 321-333 (2015).

18. Zhang, W. J. et al. Interaction of barley powdery mildew effector candidate CSEP0055 with the defence protein PR17c. Mol. Plant Pathol. 13, 1110-1119 (2012).

19. Pennington, H. G. et al. Interactions between the Powdery Mildew Effector BEC1054 and Barley Proteins Identify Candidate Host Targets. J. Proteome Res. 15, 826-839 (2016).

20. Pliego, C. et al. Host-induced gene silencing in barley powdery mildew reveals a class of ribonuclease-like effectors. Mol. Plant Microbe Interact. 26, 633-642 (2013).

21. Whigham, E. et al. Broadly conserved fungal effector BEC1019 suppresses host cell death and enhances pathogen virulence in powdery mildew of barley (Hordeum vulgare L.). Mol. Plant-Microbe Interact. 28, 968-983 (2015).

22. Ravensdale, M., Nemri, A., Thrall, P. H., Ellis, J. G. \& Dodds, P. N. Coevolutionary interactions between host resistance and pathogen effector genes in flax rust disease. Mol. Plant Pathol. 12, 93-102 (2011).

23. Dodds, P. N. et al. Direct protein interaction underlies gene-for-gene specificity and coevolution of the flax resistance genes and flax rust avirulence genes. Proc. Natl Acad. Sci. USA 103, 8888-8893 (2006).
24. Yahiaoui, N., Brunner, S. \& Keller, B. Rapid generation of new powdery mildew resistance genes after wheat domestication. Plant J. 47, 85-98 (2006)

25. Bhullar, N. K., Mackay, M. \& Keller, B. Genetic diversity of the Pm3 powdery mildew resistance alleles in wheat gene bank accessions as assessed by molecular markers. Diversity 2, 768-786 (2010).

26. Stirnweis, D. et al. Suppression among alleles encoding nucleotidebinding-leucine-rich repeat resistance proteins interferes with resistance in F1hybrid and allele-pyramided wheat plants. Plant J. 79, 893-903 (2014).

27. Seeholzer, S. et al. Diversity at the Mla powdery mildew resistance locus from cultivated barley reveals sites of positive selection. Mol. Plant-Microbe Interact. 23, 497-509 (2010).

28. Bourras, S. et al. Multiple avirulence loci and allele-specific effector recognition control the $\mathrm{Pm} 3$ race-specific resistance of wheat to powdery mildew. Plant Cell 27, 2991-3012 (2015).

29. McNally, K. E. et al. Distinct domains of the AVRPM3A2/F2 avirulence protein from wheat powdery mildew are involved in immune receptor recognition and putative effector function. New Phytol. 218, 681-695 (2018).

30. Praz, C. R. et al. AvrPm2 encodes an RNase-like avirulence effector which is conserved in the two different specialized forms of wheat and rye powdery mildew fungus. New Phytol. 213, 1301-1314 (2017).

31. Lu, X. et al. Allelic barley MLA immune receptors recognize sequenceunrelated avirulence effectors of the powdery mildew pathogen. Proc. Natl Acad. Sci. USA 113, E6486-E6495 (2016).

32. Parlange, F. et al. Genetic and molecular characterization of a locus involved in avirulence of Blumeria graminis $\mathrm{f}$. sp. tritici on wheat Pm3 resistance alleles. Fungal Genet. Biol. 82, 181-192 (2015).

33. Lipka, A. E. et al. GAPIT: genome association and prediction integrated tool Bioinformatics 28, 2397-2399 (2012).

34. Müller, M. C. et al. A chromosome-scale genome assembly reveals a highly dynamic effector repertoire of wheat powdery mildew. New Phytol. 221, 2176-2189 (2019).

35. Brunner, S. et al. Intragenic allele pyramiding combines different specificities of wheat Pm3 resistance alleles. Plant J. 64, 433-445 (2010).

36. Zimmermann, L. et al. A completely reimplemented MPI bioinformatics toolkit with a new HHpred server at its core. J. Mol. Biol. 430, 2237-2243 (2018).

37. Källberg, M. et al. Template-based protein structure modeling using the RaptorX web server. Nat. Protoc. 7, 1511-1522 (2012).

38. Praz, C. R. et al. Non-parent of origin expression of numerous effector genes indicates a role of gene regulation in host adaption of the hybrid triticale powdery mildew pathogen. Front. Plant Sci. 9, 49 (2018).

39. Rose, L. E. et al. The maintenance of extreme amino acid diversity at the disease resistance gene, RPP13, in Arabidopsis thaliana. Genetics 166, 1517-1527 (2004).

40. Bhullar, N. K., Street, K., Mackay, M., Yahiaoui, N. \& Keller, B. Unlocking wheat genetic resources for the molecular identification of previously undescribed functional alleles at the Pm3 resistance locus. Proc. Natl Acad. Sci. USA 106, 9519-9524 (2009).

41. Hall, S. A. et al. Maintenance of genetic variation in plants and pathogens involves complex networks of gene-for-gene interactions. Mol. Plant Pathol. 10, 449-457 (2009).

42. Dodds, P. N. The melampsora lini AvrL567 avirulence genes are expressed in haustoria and their products are recognized inside plant cells. Plant Cell 16, 755-768 (2004)

43. de Guillen, K. et al. Structure analysis uncovers a highly diverse but structurally conserved effector family in phytopathogenic fungi. PLoS Pathog. 11, e1005228 (2015).

44. Zhang, S. et al. Function and evolution of Magnaporthe oryzae avirulence gene AvrPib responding to the rice blast resistance gene Pib. Sci. Rep. 5, 11642 (2015).

45. Maqbool, A. et al. Structural basis of pathogen recognition by an integrated HMA domain in a plant NLR immune receptor. Elife https://doi.org/10.7554/ eLife.08709 (2015).

46. Matsumura, K. \& Tosa, Y. The rye mildew fungus carries avirulence genes corresponding to wheat genes for resistance to races of the wheat mildew fungus. Phytopathology 85, 753 (1995).

47. Menardo, F. et al. Hybridization of powdery mildew strains gives rise to pathogens on novel agricultural crop species. Nat. Genet. 48, 201-205 (2016).

48. Inoue, Y. et al. Evolution of the wheat blast fungus through functional losses in a host specificity determinant. Science 357, 80-83 (2017).

49. Tosa, Y. A model for the evolution of formae speciales and races. Phytopathology 82, 728-730 (1992).

50. Liao, J. et al. Pathogen effectors and plant immunity determine specialization of the blast fungus to rice subspecies. Elife 5, e19377 (2016). 
51. Schulze-Lefert, P. \& Panstruga, R. A molecular evolutionary concept connecting nonhost resistance, pathogen host range, and pathogen speciation. Trends in Plant Science 16, 117-125 (2011). https://doi.org/10.1016/j. tplants.2011.01.001

52. Maekawa, T., Kracher, B., Vernaldi, S., Ver Loren van Themaat, E. \& SchulzeLefert, P. Conservation of NLR-triggered immunity across plant lineages. Proc. Natl Acad. Sci. USA 109, 20119-20123 (2012).

53. Parlange, F. et al. A major invasion of transposable elements accounts for the large size of the Blumeria graminis f.sp. tritici genome. Funct. Integr. Genomics 11, 671-677 (2011).

54. Zeng, F. S. et al. Virulence and diversity of Blumeria graminis f. sp. tritici populations in China. J. Integr. Agric. 13, 2424-2437 (2014).

55. Gong, S. et al. An approach for long-term preservation of Blumeria graminis $\mathrm{f}$. sp. tritici. Trop. Plant Pathol. 40, 127-133 (2015).

56. Brunner, S. et al. Transgenic Pm $3 \mathrm{~b}$ wheat lines show resistance to powdery mildew in the field. Plant Biotechnol. J. 9, 897-910 (2011).

57. Brunner, S. et al. Transgenic Pm3 multilines of wheat show increased powdery mildew resistance in the field. Plant Biotechnol. J. 10, 398-409 (2012).

58. Thordal-Christensen, H., Zhang, Z., Wei, Y. \& Collinge, D. B. Subcellular localization of $\mathrm{H} 2 \mathrm{O} 2$ in plants. $\mathrm{H} 2 \mathrm{O} 2$ accumulation in papillae and hypersensitive response during the barley-powdery mildew interaction. Plant J. 11, 1187-1194 (1997).

59. Nei, M. \& Gojobori, T. Simple methods for estimating the numbers of synonymous and nonsynonymous nucleotide substitutions. Mol. Biol. Evol. 3, 418-426 (1986).

60. Rozas, J. et al. DnaSP 6: DNA sequence polymorphism analysis of large data sets. Mol. Biol. Evol. 34, 3299-3302 (2017).

61. Petersen, T. N., Brunak, S., Von Heijne, G. \& Nielsen, H. SignalP 4.0: discriminating signal peptides from transmembrane regions. Nat. Methods $\mathbf{8}$, 785-786 (2011).

62. Himmelbach, A. et al. A set of modular binary vectors for transformation of cereals. Plant Physiol. 145, 1192-1200 (2007).

63. Frenkel, Z., Paux, E., Mester, D., Feuillet, C. \& Korol, A. LTC: a novel algorithm to improve the efficiency of contig assembly for physical mapping in complex genomes. BMC Bioinform. 11, 584 (2010).

64. Robinson, M. D., McCarthy, D. J. \& Smyth, G. K. edgeR: a Bioconductor package for differential expression analysis of digital gene expression data. Bioinformatics 26, 139-140 (2010).

65. Dobin, A. et al. STAR: Ultrafast universal RNA-seq aligner. Bioinformatics 29, 15-21 (2013).

66. Patro, R., Duggal, G., Love, M. I., Irizarry, R. A. \& Kingsford, C. Salmon provides fast and bias-aware quantification of transcript expression. Nat. Methods 14, 417-419 (2017).

67. Langmead, B. \& Salzberg, S. L. Fast gapped-read alignment with Bowtie 2. Nat. Methods 9, 357-359 (2012).

68. Li, H. et al. The Sequence Alignment/Map format and SAMtools. Bioinformatics 25, 2078-2079 (2009).

69. Garrison, E. \& Marth, G. Haplotype-based variant detection from shortread sequencing. Preprint at https://arxiv.org/abs/1207.3907 (2012).

\section{Acknowledgements}

We would like to acknowledge Dr. Kohji Nishimura (Shimane University, Japan) for technical support with protein expression assays. We would like to acknowledge the technical support from Gerhard Herren, Helen Zbinden, Karl Huwiler, Gabriele Buchmann and Ritta Rabbat. We would like to acknowledge the financial support of the Swiss National Science Foundation (SNSF) grants 310030_163260 and 310030B_182833, the European Research Council (ERC), and the University Research Priority Program (URPP) of the University of Zurich.

\section{Author contributions}

S.B., L.K., and B.K. wrote the paper. S.B., D.Y. and B.K. coordinated the research. S.B., L.K., M.X., D.Y. and B.K. designed the research. S.B., L.K., C.K., M.S., P.A., S.F., F.P., L.K. S., V.W., S.L. and J.I. performed lab experiments. S.B., L.K., M.X., C.R.P., M.C.M., F.P. and R.B.D. performed genetic analyses. C.R.P., M.C.M., F.M., S.R., S.O. and T.W. performed bioinformatics analyses. S.B., C.R.P. and F.M. developed the effector benchmarking approach. M.X., C.R.P., M.C.M. and F.M. developed the GWAS approach. S.B L.K., C.K., M.S. and S.F. developed and executed the AVR screens in N. benthamiana.

\section{Additional information}

Supplementary Information accompanies this paper at https://doi.org/10.1038/s41467019-10274-1.

Competing interests: The authors declare no competing interests.

Reprints and permission information is available online at http://npg.nature.com/ reprintsandpermissions/

Journal peer review information: Nature Communications thanks Yukio Tosa and other anonymous reviewer(s) for their contribution to the peer review of this work.

Publisher's note: Springer Nature remains neutral with regard to jurisdictional claims in published maps and institutional affiliations.

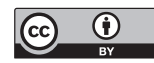

Open Access This article is licensed under a Creative Commons Attribution 4.0 International License, which permits use, sharing, adaptation, distribution and reproduction in any medium or format, as long as you give appropriate credit to the original author(s) and the source, provide a link to the Creative Commons license, and indicate if changes were made. The images or other third party material in this article are included in the article's Creative Commons license, unless indicated otherwise in a credit line to the material. If material is not included in the article's Creative Commons license and your intended use is not permitted by statutory regulation or exceeds the permitted use, you will need to obtain permission directly from the copyright holder. To view a copy of this license, visit http://creativecommons.org/ licenses/by/4.0/.

(c) The Author(s) 2019 